\title{
Snapshots of Conformal Field Theory
}

\author{
Katrin Wendland
}

\begin{abstract}
In snapshots, this exposition introduces conformal field theory, with a focus on those perspectives that are relevant for interpreting superconformal field theory by Calabi-Yau geometry. It includes a detailed discussion of the elliptic genus as an invariant which certain superconformal field theories share with the CalabiYau manifolds. K3 theories are (re)viewed as prime examples of superconformal field theories where geometric interpretations are known. A final snapshot addresses the K3-related Mathieu Moonshine phenomena, where a lead role is predicted for the chiral de Rham complex.
\end{abstract}

\section{Introduction}

Conformal quantum field theory (CFT) became popular in physics thanks to the work by Belavin, Polyakov and Zamolodchikov. In their seminal paper [BPZ84], on the one hand, they lay the mathematical foundations of axiomatic CFT, and on the other hand, they show the physical significance of CFT for surface phenomena in statistical physics by describing certain phase transitions of second order through CFT.

Another common source of conformal field theories is string theory, which is many theoreticians' favorite candidate for the unification of all interactions, including gravity. Here, particles are described by strings that move in some potentially complicated background geometry. The string dynamics are governed by a so-called non-linear sigma model, such that conformal invariance yields the string equations of motion. The quantum field theory living on the worldsheet of the string then is a CFT. This implies deep relations between CFT and geometry, which have already

Katrin Wendland

Mathematics Institute, Freiburg University, Eckerstr. 1, 79104 Freiburg i. Br., Germany,

e-mail: katrin.wendlandemath.uni-freiburg.de 
led to a number of intriguing insights in geometry, demanding for a more resilient bridge between mathematics and physics.

For example, in the early 90 s mirror symmetry provided a first success story for the interaction between mathematics and physics in the context of CFT [LVW89, GP90, CdGP91, CLS90]. However, a rigorous approach to those types of CFTs that are relevant for such deep insights in algebraic geometry was not available, at the time. As a result, the interaction between mathematics and physics in many cases amounted to a rather imbalanced division of work, where theoretical physicists provided the most amazing predictions and left them to the mathematicians for a proof, who in turn successfully detached their theories from their origins in physics.

With the advent of Monstrous Moonshine [Tho79a, Tho79b, CN79, FLM84, Bor86, Gan06], and with Borcherds' Fields Medal in 1998 "for his contributions to algebra, the theory of automorphic forms, and mathematical physics, including the introduction of vertex algebras and Borcherds' Lie algebras, the proof of the Conway-Norton moonshine conjecture and the discovery of a new class of automorphic infinite products" [Man98], the subject of conformal field theory, per se, began to become more popular in mathematics. Indeed, the comparatively new notion of vertex algebras provided a rigorous mathematical foundation to the most basic ingredients of conformal quantum field theory and thereby offered a viable approach to CFT for mathematicians. Nevertheless, the quest to fill the gap between abstract mathematical approaches to CFT and those types of models that are of interest in physics, and that are relevant for deeper insights in algebraic and enumerative geometry, has not yet been completed. The present work attempts to make a contribution to this quest.

Since this exposition can certainly only provide some snapshots of CFT, it has to follow a subjective selection and presentation of material. The guiding principle is the conviction that on the one hand, the foundation of the discussion has to be a mathematically rigorous definition of CFT, which is independent of string theory, while on the other hand, those predictions from CFT which affect the geometry of Calabi-Yau manifolds are among the most intriguing ones. To state and understand the latter, one needs to work with a mathematical formulation of CFT which allows to make contact with the non-linear sigma models in physics, thus sadly excluding a number of popular approaches to CFT. Moreover, the discussion is restricted to so-called two-dimensional Euclidean unitary CFTs.

In more detail, this work is structured as follows:

Section 2 provides a definition of some of the ingredients of CFT. The conformal vertex algebras serve as our point of entry in Section 2.1 since this part of CFT is probably the most natural for mathematicians. We proceed in Section 2.2 by listing the crucial ingredients that underlie a definition of superconformal field theory, along with additional required properties. The presentation makes no claim for completeness, but according to our declared conviction, we focus on those aspects that are relevant for the discussion of geometric interpretations as introduced later. This in particular restricts our attention to the so-called $N=(2,2)$ superconformal 
field theories with space-time supersymmetry. A useful class of examples, which is well understood, is given by the toroidal $N=(2,2)$ superconformal field theories presented in Section 2.3. We summarize the definition and properties of the chiral de Rham complex in Section 2.5, as an example of a sheaf of conformal vertex algebras on an arbitrary smooth algebraic variety, which thus provides a link between standard ingredients of CFT and geometric quantities. Since this link is not entirely understood to the very day, for clarification, our discussion rests on the special role of the elliptic genus as an invariant that certain superconformal field theories share with the Calabi-Yau manifolds 1 , as is discussed in some detail in Section 2.4

The elliptic genus is also crucial for our definition of $\mathrm{K} 3$ theories in Section 3. This class of CFTs deserves some attention, as it provides the only examples of non-linear sigma models on Calabi-Yau manifolds other than tori, where at least there are precise predictions on the global form of the moduli space, implying some very explicit relations between quantities in geometry and CFT. We motivate the definition of $\mathrm{K} 3$ theories in detail, and we summarize some of the known properties of these theories. In particular, Proposition 2 recalls the dichotomy of $N=(2,2)$ superconformal field theories at central charges $c=6, \bar{c}=6$ with space-time supersymmetry and integral $U(1)$-charges. Indeed, these theories fall into two classes, namely the toroidal and the K3 theories. Thus Proposition 2 is the conformal field theoretic counterpart of the classification of Calabi-Yau 2-manifolds into complex two-tori, on the one hand, and K3 surfaces, on the other. Our proof [Wen00, §7.1], which is little known, is summarized in the Appendix.

The final Section 4 is devoted to recent developments in the study of K3 theories, related to the mysterious phenomena known as Mathieu Moonshine. We recall the route to discovery of these phenomena, which also proceeds via the elliptic genus. We offer some ideas towards a geometric interpretation, arguing that one should expect the chiral de Rham complex to be crucial in unraveling the Mathieu Moonshine mysteries. The section closes with an open conjecture, which is related to Mathieu Moonshine, which however is formulated neither alluding to moonshine nor to CFT, and which therefore is hoped to be of independent interest.

\section{Ingredients of conformal field theory}

The present section collects ingredients of conformal field theory (CFT), more precisely of TWO-DIMENSIONAL EUCLIDEAN UNITARY CONFORMAL FIELD THEORY. These adjectives translate into the properties of the underlying quantum field theory as follows: first, all fields are parametrized on a TWO-DIMENSIONAL WORLDSHEET, which comes equipped with a EUCLIDEAN METRIC. Second, the fields transform covariantly under CONFORMAL MAPS between such worldsheets. Furthermore, the space of states in such a CFT is equipped with a positive defi-

\footnotetext{
${ }^{1}$ Disclaimer: in this work, all Calabi-Yau manifolds are compact, by definition.
} 
nite metric, with respect to which the infinitesimal conformal transformations act UNITARILY.

We begin by describing the simplest fields in our CFTs in terms of the so-called VERTEX ALGEBRAS in Section 2.1 Next, Section 2.2 summarizes a definition of conformal field theory, with the toroidal conformal field theories presented as a class of examples in Section 2.3. In Sections 2.4 and 2.5 the related notions of the elliptic genus and the chiral de Rham complex are discussed in the context of superconformal field theories. As such, the present section collects ingredients of CFT, with a focus on some of those ingredients that are under investigation to the very day.

\subsection{Conformal and superconformal vertex algebras}

We begin by recalling the notion of FIELDS, following [Kac98]. The theory is built on the earlier results [LW78, FK81, Bor86], see also [FBZ04] for a very readable exposition. This definition is most convenient, because it naturally implements the representation theory inherent to CFTs. As we shall see at the end of this section, for the chiral states of CFTs it also allows a straightforward definition of the $n$-point functions.

\section{Definition 1.}

Consider a $\mathbb{C}$-vectorspace $\mathbb{H}$.

- $\mathbb{H}\left[\left[z_{1}^{ \pm 1}, \ldots, z_{n}^{ \pm 1}\right]\right]$ denotes the vectorspace of formal power series

$$
v\left(z_{1}, \ldots, z_{n}\right)=\sum_{i_{1}, \ldots, i_{n} \in \mathbb{Z}} \widehat{v}_{i_{1}, \ldots, i_{n}} z_{1}^{i_{1}} \cdots z_{n}^{i_{n}}, \quad \widehat{v}_{i_{1}, \ldots, i_{n}} \in \mathbb{H} .
$$

- For $A \in \operatorname{End}_{\mathbb{C}}(\mathbb{H})\left[\left[z_{1}^{ \pm 1}, \ldots, z_{n}^{ \pm 1}\right]\right]$, and for $\alpha \in \mathbb{H}^{*}:=\operatorname{Hom}_{\mathbb{C}}(\mathbb{H}, \mathbb{C})$ and $v \in$ $\mathbb{H}$, we set

$$
\left\langle\alpha, A\left(z_{1}, \ldots, z_{n}\right) v\right\rangle:=\sum_{i_{1}, \ldots, i_{n} \in \mathbb{Z}}\left\langle\alpha, \widehat{A}_{i_{1}, \ldots, i_{n}} v\right\rangle z_{1}^{i_{1}} \cdots z_{n}^{i_{n}} \in \mathbb{C}\left[\left[z_{1}^{ \pm 1}, \ldots, z_{n}^{ \pm 1}\right]\right],
$$

where on the right hand side, $\langle\cdot, \cdot\rangle$ denotes the natural pairing between $\mathbb{H}^{*}$ and $\mathbb{H}$.

- If $\left.A(z) \in \operatorname{End}_{\mathbb{C}}(\mathbb{H})\left[z^{ \pm 1}\right]\right]$ with $A(z)=\sum_{n} \widehat{A}_{n} z^{n}$, then $\partial A$ denotes the formal derivative of $A$,

$$
\partial A(z)=\sum_{n \in \mathbb{Z}} n \widehat{A}_{n} z^{n-1} \quad \in \operatorname{End}_{\mathbb{C}}(\mathbb{H})\left[\left[z^{ \pm 1}\right]\right] .
$$

- A formal power series $\left.A(z) \in \operatorname{End}_{\mathbb{C}}(\mathbb{H})\left[z^{ \pm 1}\right]\right]$ is called a FIELD on $\mathbb{H}$ if $A(z)=\sum_{n} \widehat{A}_{n} z^{n}$ obeys 


$$
\forall v \in \mathbb{H}: \quad \exists N \in \mathbb{Z} \text { such that } \widehat{A}_{n} v=0 \forall n<N .
$$

The endomorphisms $\widehat{A}_{n}$ are called the MODES of the field $A$.

In other words, if $A$ is a field on $\mathbb{H}$, then for every $v \in \mathbb{H}$ the expression $A(z) v=\sum_{n}\left(\widehat{A}_{n} v\right) z^{n}$ is a formal Laurent series with coefficients in $\mathbb{H}$ and with only finitely many non-zero contributions $\left(\widehat{A}_{n} v\right) z^{n}$ with $n<0$. In the context of CFTs one can introduce a completion $\overline{\mathbb{H}}$ of $\mathbb{H}$ with respect to an appropriate topology and then for every $z \in \mathbb{C}^{*}$ view $A(z)$ as a linear operator from $\mathbb{H}$ to $\mathbb{H}$, see for example [FBZ04, §1.2.1]. Accordingly, we call a field $A(z)=\sum_{n} \widehat{A}_{n} z^{n}$ CONSTANT if $\widehat{A}_{n}=0$ for all $n \neq 0$. Similarly, if for $v \in \mathbb{H}$ we have $\widehat{A}_{n} v=0$ for all $n<0$, then we say that $A(z) v$ IS WELL-DEFINED IN $z=0$, and $A(z) v_{\mid z=0}=\widehat{A}_{0} v$. Note that a field $A$ according to Definition 1 can be viewed as an operator valued distribution, as usual in quantum field theory. Indeed, by means of the residue, $A(z)$ yields a linear map from complex polynomials into $\mathbb{H}$. By definition, the space $\mathbb{H}$ carries a representation of the Lie algebra generated by the modes of every field on $\mathbb{H}$, with the Lie bracket that is inherited from $\operatorname{End}_{\mathbb{C}}(\mathbb{H})$, namely the commutator.

Let us now consider two fields $A, B$ on $\mathbb{H}$. While the expressions $A(z) B(w)$ and $B(w) A(z)$ make sense as formal power series in $\left.\operatorname{End}_{\mathbb{C}}(\mathbb{H})\left[z^{ \pm 1}, w^{ \pm 1}\right]\right]$, a priori it is impossible to interpret them as fields. In general, we expect singular behavior for the coefficients when we insert $w=z$, and in fact the form of this singularity captures the most important aspects of CFT. Here, the notions of LOCALITY and NORMAL ORDERED PRODUCTS come to aid:

\section{Definition 2.}

1. Let $\partial_{w}$ denote the formal derivative with respect to $w$ in $\mathbb{C}\left[\left[z^{ \pm 1}, w^{ \pm 1}\right]\right]$. On $\mathbb{C}[[z, w]]\left[z^{-1}, w^{-1},(z-w)^{-1}\right]$, we define the $\mathbb{C}[[z, w]]\left[z^{-1}, w^{-1}\right]$-linear operators $\boldsymbol{l}_{z>w}$ and $\boldsymbol{l}_{w>z}$ into $\left.\mathbb{C}\left[z^{ \pm 1}, w^{ \pm 1}\right]\right]$ with

$$
\text { for } k \in \mathbb{N}: \quad \begin{aligned}
l_{z>w}\left(k !(z-w)^{-k-1}\right) & =\partial_{w}^{k} \frac{1}{z} \sum_{n=0}^{\infty}\left(\frac{w}{z}\right)^{n}, \\
& l_{w>z}\left(k !(z-w)^{-k-1}\right)=-\partial_{w}^{k} \frac{1}{w} \sum_{n=0}^{\infty}\left(\frac{z}{w}\right)^{n} .
\end{aligned}
$$

2. Fields $A, B$ on $\mathbb{H}$ are called LOCAL WITH RESPECT TO EACH OTHER if there exist a so-called NORMAL ORDERED PRODUCT $: A(z) B(w): \in$ $\left.\operatorname{End}_{\mathbb{C}}(\mathbb{H})\left[z^{ \pm 1}, w^{ \pm 1}\right]\right]$ and fields $X_{0}, \ldots, X_{N-1}$ and $: A B$ : on $\mathbb{H}$, such that for every $\alpha \in \mathbb{H}^{*}$ and $v \in \mathbb{H}$,

- we have $\langle\alpha,: A(z) B(w): v\rangle \in \mathbb{C}[[z, w]]\left[z^{-1}, w^{-1}\right]$,

- in $\operatorname{End}_{\mathbb{C}}(\mathbb{H})\left[z^{ \pm 1}\right]$, we have $: A B:(z)=: A(z) B(w):\left.\right|_{w=z}$, 
- in $\mathbb{C}[[z, w]]\left[z^{-1}, w^{-1},(z-w)^{-1}\right]$, we have

$$
\begin{aligned}
& \langle\alpha, A(z) B(w) v\rangle=\imath_{z>w}\left(\sum_{j=0}^{N-1} \frac{\left\langle\alpha, X_{j}(w) v\right\rangle}{(z-w)^{j+1}}\right)+\langle\alpha,: A(z) B(w): v\rangle, \\
& \langle\alpha, B(w) A(z) v\rangle=\imath_{w>z}\left(\sum_{j=0}^{N-1} \frac{\left\langle\alpha, X_{j}(w) v\right\rangle}{(z-w)^{j+1}}\right)+\langle\alpha,: A(z) B(w): v\rangle .
\end{aligned}
$$

As a shorthand notation one writes the so-called OPERATOR PRODUCT EXPANSION (OPE)

$$
A(z) B(w) \sim \sum_{j=0}^{N-1} \frac{X_{j}(w)}{(z-w)^{j+1}},
$$

where contributions that are regular at $z=w$ may be omitted on the right hand side at will.

For the special fields that feature in CFTs, the formal power series in the above definition yield convergent functions in complex variables $z$ and $w$ on appropriate domains in $\mathbb{C}$. Then the operators $l_{z>w}$ and $\boldsymbol{l}_{w>z}$ implement the Taylor expansions about $z=w$ in the domains $|z|>|w|$ and $|w|>|z|$, respectively. We therefore refer to these operators as (FORMAL) TAYLOR EXPANSIONS. The OPE thus captures the singular behavior of the expressions $\langle\alpha, A(z) B(w) v\rangle$ when $z \sim w$, where locality of the fields $A$ and $B$ with respect to each other restricts the possible singularities to poles at $z=w$. For $[A(z), B(w)]:=A(z) B(w)-B(w) A(z) \in \operatorname{End}_{\mathbb{C}}(\mathbb{H})\left[\left[z^{ \pm 1}, w^{ \pm 1}\right]\right.$, the observation that, in general, $\langle\alpha,[A(z), B(w)] v\rangle$ does not vanish, accounts for the fact that $(z-w)^{-1}$ and its derivatives have different Taylor expansions in the domains $|z|>|w|$ and $|w|>|z|$, respectively. Hence the modes of the fields $X_{j}$ in the OPE encode the commutators $\left[\widehat{A}_{n}, \widehat{B}_{m}\right]$ of the modes of $A$ and $B$.

The Definition 2 of the normal ordered product : $A(z) B(w)$ : of two fields $A, B$ on $\mathbb{H}$ yields $: A(z) B(w):=A_{+}(z) B(w)+B(w) A_{-}(z)$ if $A(z)=A_{+}(z)+A_{-}(z)$, where $A_{+}(z):=\sum_{n \geq 0} \widehat{A}_{n} z^{n}$ and $A_{-}(z):=\sum_{n<0} \widehat{A}_{n} z^{n}$. Hence our definition of normal ordered product amounts to a choice in decomposing $A(z)=A_{+}(z)+A_{-}(z)$ as stated, which accrues from the choice of decomposing the formal power series

$$
\sum_{m=-\infty}^{\infty} z^{m} w^{-m-1}=l_{z>w}\left((z-w)^{-1}\right)-l_{w>z}\left((z-w)^{-1}\right) \quad \in \mathbb{C}\left[\left[z^{ \pm 1}, w^{ \pm 1}\right]\right] .
$$

In the context of superconformal field theories, these notions are generalized to include ODD fields; if both $A$ and $B$ are odd, then locality amounts to

$$
\langle\alpha, A(z) B(w) v\rangle=l_{z>w}\left(\sum_{j=0}^{N-1} \frac{\left\langle\alpha, X_{j}(w) v\right\rangle}{(z-w)^{j+1}}\right)+\langle\alpha,: A(z) B(w): v\rangle,
$$




$$
-\langle\alpha, B(w) A(z) v\rangle=\imath_{w>z}\left(\sum_{j=0}^{N-1} \frac{\left\langle\alpha, X_{j}(w) v\right\rangle}{(z-w)^{j+1}}\right)+\langle\alpha,: A(z) B(w): v\rangle,
$$

abbreviated by the same OPE as in Definition 2 , and the bracket $[\cdot, \cdot]$ in the above argument is replaced by a superbracket with $[A(z), B(w)]=A(z) B(w)+B(w) A(z)$ for odd fields $A, B$. The space $\mathbb{H}$, accordingly, furnishes a representation of the superLie algebra generated by the modes of the fields on $\mathbb{H}$.

The following list of examples provides some basic fields in the simplest CFTs:

Example 1. [U(1)-current]

We consider the complex Lie algebra $\mathscr{A}$ with $\mathbb{C}$-vectorspace basis $\left\{C ; a_{n}, n \in \mathbb{Z}\right\}$, where $C$ is a central element and the Lie bracket obeys

$$
\forall m, n \in \mathbb{Z}: \quad\left[a_{n}, a_{m}\right]=m \delta_{n+m, 0} \cdot \frac{C}{3} .
$$

Choose some $c \in \mathbb{R}$ and let $\mathbb{H}$ denote the $\mathscr{A}$-module which under the $\mathscr{A}$-action is generated by a single non-zero vector $\Omega$, with submodule of relations generated by

$$
a_{n} \Omega=0 \quad \forall n \leq 0, \quad C \Omega=c \Omega .
$$

The space $\mathbb{H}$ can be viewed as polynomial ring in the $a_{n}$ with $n>0$. One then checks that the so-called $U(1)$-CURRENT

$$
J(z):=\sum_{n=-\infty}^{\infty} a_{n} z^{n-1}
$$

is a well-defined field on $\mathbb{H}$ which obeys the OPE

$$
J(z) J(w) \sim \frac{c / 3}{(z-w)^{2}} .
$$

In particular, $J$ is local with respect to itself. Here and in the following, a constant field which acts by multiplication by $\Lambda \in \mathbb{C}$ on $\mathbb{H}$ is simply denoted by $\Lambda$.

Example 2. [ Virasoro field]

For the $U(1)$-current $J$ on the vectorspace $\mathbb{H}$ introduced in the previous example, assume $c \neq 0$ and let $T(z):=\frac{3}{2 c}: J J:(z)$. One checks that with $c^{\bullet}=1$ this field on $\mathbb{H}$ obeys the OPE

$$
T(z) T(w) \sim \frac{c^{\bullet} / 2}{(z-w)^{4}}+\frac{2 T(w)}{(z-w)^{2}}+\frac{\partial T(w)}{z-w},
$$

which for the modes of $T(z)=\sum_{n} L_{n} z^{n-2}$ translates into

$$
\forall n, m \in \mathbb{Z}: \quad\left[L_{n}, L_{m}\right]=(m-n) L_{m+n}+\delta_{n+m, 0} \frac{c^{\bullet}}{12} m\left(m^{2}-1\right) .
$$


The above equation (2) defines the Virasoro ALgebra at central charge $c^{\bullet}$, whose underlying vectorspace has $\mathbb{C}$-vectorspace basis $\left\{c^{\bullet} ; L_{n}, n \in \mathbb{Z}\right\}$. This Lie algebra is the central extension by $\operatorname{span}_{\mathbb{C}}\left\{c^{\bullet}\right\}$ of the Lie algebra of infinitesimal conformal transformations of the punctured Euclidean plane $\mathbb{C}^{*}$.

Example 3. [bc- $\beta \gamma$-system]

Let $D \in \mathbb{N}$, and consider the super-Lie algebra $\mathscr{A}_{D}$ with $\mathbb{C}$-vectorspace basis $\{C$; $\left.a_{n}^{i}, b_{n}^{i}, \varphi_{n}^{i}, \psi_{n}^{i}, n \in \mathbb{Z}, i \in\{1, \ldots, D\}\right\}$, where the $a_{n}^{i}, b_{n}^{i}$ and the central element $C$ are even, while the $\varphi_{n}^{i}, \psi_{n}^{i}$ are odd, and the only non-vanishing basic super-Lie brackets are

$$
\forall m, n \in \mathbb{Z}, i, j \in\{1, \ldots, D\}: \begin{aligned}
{\left[a_{n}^{i}, b_{m}^{j}\right] } & =\delta^{i, j} \delta_{n+m, 0} \cdot C, \\
\left\{\psi_{n}^{i}, \varphi_{m}^{j}\right\} & =\delta^{i, j} \delta_{n+m, 0} \cdot C .
\end{aligned}
$$

Here, $\{\cdot, \cdot\}$ denotes the super-Lie bracket between odd elements of $\mathscr{A}_{D}$, as is customary in the physics literature. Let $\mathbb{H}$ denote the $\mathscr{A}_{D}$-module which under the $\mathscr{A}_{D^{-}}$ action is generated by a single non-zero vector $\Omega$, with submodule of relations generated by

$$
\begin{aligned}
\forall n \leq 0, m<0, i, j \in\{1, \ldots, D\}: & a_{n}^{i} \Omega=0, b_{m}^{j} \Omega=0, \\
& \psi_{n}^{i} \Omega=0, \varphi_{m}^{j} \Omega=0 ; \quad C \Omega=\Omega .
\end{aligned}
$$

Generalizing Examples 1 and 2 above, one checks that

$$
\begin{aligned}
a^{i}(z) & :=\sum_{n=-\infty}^{\infty} a_{n}^{i} z^{n-1}, \quad b^{i}(z):=\sum_{m=-\infty}^{\infty} b_{m}^{i} z^{m} \\
\psi^{i}(z) & :=\sum_{n=-\infty}^{\infty} \psi_{n}^{i} z^{n-1}, \quad \varphi^{i}(z):=\sum_{m=-\infty}^{\infty} \varphi_{m}^{i} z^{m}, \quad i \in\{1, \ldots, D\}
\end{aligned}
$$

defines pairwise local fields $a^{i}, b^{i}, \psi^{i}, \varphi^{i}$ on $\mathbb{H}$. Moreover, one finds the OPEs

$$
a^{i}(z) b^{j}(w) \sim \frac{\delta^{i, j}}{z-w}, \quad \varphi^{i}(z) \psi^{j}(w) \sim \frac{\delta^{i, j}}{z-w} \quad \forall i, j \in\{1, \ldots, D\},
$$

while all other basic OPEs vanish, and the field

$$
T^{\mathrm{top}}(z):=\sum_{j=1}^{D}\left(: \partial b^{j} a^{j}:(z)+: \partial \varphi^{j} \psi^{j}:(z)\right)
$$

is a Virasoro field obeying (1) at central charge $c^{\bullet}=0$.

Example 4. [topological $N=2$ superconformal algebra]

With $\mathscr{A}_{D}, \mathbb{H}$, and the fields of the $b c-\beta \gamma$-system defined in the above Example 3 , let

$$
J(z):=\sum_{j=1}^{D}: \varphi^{j} \psi^{j}:(z), Q(z):=\sum_{j=1}^{D}: a^{j} \varphi^{j}:(z), G(z):=\sum_{j=1}^{D}: \psi^{j} \partial b^{j}:(z) .
$$


These fields obey the so-called TOPOLOGICAL $N=2$ SUPERCONFORMAL ALGEBRA at central charge $c=3 D$ :

$$
\begin{aligned}
T^{\mathrm{top}}(z) T^{\mathrm{top}}(w) & \sim \frac{2 T^{\mathrm{top}}(w)}{(z-w)^{2}}+\frac{\partial T^{\mathrm{top}}(w)}{z-w}, \\
T^{\mathrm{top}}(z) J(w) & \sim-\frac{c / 3}{(z-w)^{3}}+\frac{J(w)}{(z-w)^{2}}+\frac{\partial J(w)}{z-w}, \quad J(z) J(w) \sim \frac{c / 3}{(z-w)^{2}} \\
T^{\mathrm{top}}(z) Q(w) & \sim \frac{Q(w)}{(z-w)^{2}}+\frac{\partial Q(w)}{z-w}, \quad Q(z) Q(w) \sim 0, \quad J(z) Q(w) \sim \frac{Q(w)}{z-w}, \\
T^{\mathrm{top}}(z) G(w) & \sim \frac{2 G(w)}{(z-w)^{2}}+\frac{\partial G(w)}{z-w}, \quad G(z) G(w) \sim 0, \quad J(z) G(w) \sim-\frac{G(w)}{z-w} \\
Q(z) G(w) & \sim \frac{c / 3}{(z-w)^{3}}+\frac{J(w)}{(z-w)^{2}}+\frac{T^{\mathrm{top}}(w)}{z-w} .
\end{aligned}
$$

Example 5. [ $N=2$ superconformal algebra]

Consider a $\mathbb{C}$-vectorspace $\mathbb{H}$ and pairwise local fields $T^{\text {top }}(z), J(z), Q(z), G(z)$ on $\mathbb{H}$ which obey the topological $N=2$ superconformal algebra (6)-(7) at central charge $c$. Now let

$$
T(z):=T^{\mathrm{top}}(z)-\frac{1}{2} \partial J(z), \quad G^{+}(z):=Q(z), \quad G^{-}(z):=G(z) .
$$

Then $T(z)$ is another Virasoro field as in (1), but now with central charge $c^{\bullet}=c$, and the fields $T(z), J(z), G^{+}(z), G^{-}(z)$ on $\mathbb{H}$ obey the so-called $N=2$ SUPERCONFORMAL ALGEBRA at central charge $c$,

$$
\begin{aligned}
T(z) T(w) & \sim \frac{c / 2}{(z-w)^{4}}+\frac{2 T(w)}{(z-w)^{2}}+\frac{\partial T(w)}{z-w}, \\
T(z) J(w) & \sim \frac{J(w)}{(z-w)^{2}}+\frac{\partial J(w)}{z-w}, \quad J(z) J(w) \sim \frac{c / 3}{(z-w)^{2}} \\
T(z) G^{ \pm}(w) & \sim \frac{3 / 2 G^{ \pm}(w)}{(z-w)^{2}}+\frac{\partial G^{ \pm}(w)}{z-w}, \quad J(z) G^{ \pm}(w) \sim \pm \frac{G^{ \pm}(w)}{z-w}, \\
G^{ \pm}(z) G^{\mp}(w) & \sim \frac{c / 3}{(z-w)^{3}} \pm \frac{J(w)}{(z-w)^{2}}+\frac{T(w) \pm \frac{1}{2} \partial J(w)}{z-w}, G^{ \pm}(z) G^{ \pm}(w) \sim 0 .
\end{aligned}
$$

Equation (8) is referred to by the statement that the fields $T^{\text {top }}(z), J(z), Q(z), G(z)$ are obtained from the fields $T(z), J(z), G^{+}(z), G^{-}(z)$ by a TOPOLOGICAL ATWIST. Analogously, fields $T^{\mathrm{top}}(z),-J(z), Q(z), G(z)$ which obey a topological $N=2$ superconformal algebra at central charge $c$ are obtained from fields $T(z), J(z)$, $G^{+}(z), G^{-}(z)$ which obey an $N=2$ superconformal algebra at central charge $c$ by a TOPOLOGICAL B-TWIST iff $T^{\mathrm{top}}(z)=T(z)-\frac{1}{2} \partial J(z), Q(z)=G^{-}(z), G=$ $G^{+}(z)$, see [Wit88b, EY90]. On the level of the $N=2$ superconformal algebras, the transition between topological A-twist and topological B-twist is induced by 
$\left(T, J, G^{+}, G^{-}\right) \mapsto\left(T,-J, G^{-}, G^{+}\right)$, an automorphism of the superconformal algebra. This automorphism is at the heart of MIRROR SYMMETRY [LVW89].

We are now ready to define one of the fundamental ingredients of CFT, namely the notion of CONFORMAL VERTEX ALGEBRA. The definition is taken from [FBZ04] and follows [FKRW95] and [Bor86, Kac98]:

Definition 3. A CONFORMAL VERTEX ALgEbRA AT CENTRAL CHARGE $c \in$ $\mathbb{C}$ is given by the following data:

- a $\mathbb{Z}$-graded $\mathbb{C}$-vectorspace $W=\oplus_{m \in \mathbb{Z}} W_{m}$ called the SPACE OF STATES,

- a special vector $\Omega \in W_{0}$ called the VACUUM,

- a linear operator $L: W \rightarrow W$ called the TRANSLATION OPERATOR,

- a special vector $T \in W_{2}$ called the CONFORMAL VECTOR,

- a linear map

$$
\left.Y(\cdot, z): W \longrightarrow \operatorname{End}(W)\left[z^{ \pm 1}\right]\right]
$$

called the STATE-FIELD CORRESPONDENCE, which assigns to every $A \in W$ a field $A(z):=Y(A, z)$ on $W$.

These data obey the following axioms:

- The VACUUm AXIOM. We have $\Omega(z)=1$, and for every $A \in W$ and $A(z)=$ $\sum_{n} \widehat{A}_{n} z^{n}$, we obtain $A(z) \Omega \in W[[z]]$, such that $A(z) \Omega$ is well-defined in $z=0$ and

$$
A(z) \Omega_{\mid z=0}=\widehat{A}_{0} \Omega=A \in W .
$$

One says: the field $A(z)$ CREATES the state $A$ from the vacuum.

- The TRANSLATION AXIOM. We have

$$
L \Omega=0 \quad \text { and } \quad \forall A \in W: \quad[L, A(z)]=\partial A(z) .
$$

- The LOCALITy AXIOM.

All fields $A(z)$ with $A \in W$ are local with respect to each other.

The (ungraded) vectorspace $W$ with $\Omega, L$, and the map $Y$ is called a VERTEX ALGEBRA. In a CONFORMAL vertex algebra, in addition

- The field $T(z)=\sum_{n=-\infty}^{\infty} L_{n} z^{n-2}$ associated to the conformal vector $T$ by the state-field correspondence is a Virasoro field obeying the OPE (1) with central charge $c^{\bullet}=c$.

- The translation operator $L$ is given by $L=L_{1}$ and has degree 1 .

- For all $m \in \mathbb{Z}, L_{0 \mid W_{m}}=m$, and for $A \in W_{m}$, the field $A(z)$ has weight $m$, i.e. $A(z)=\sum_{n=-\infty}^{\infty} A_{n} z^{n-m}$ with $A_{n} \in \operatorname{End}(W)$ of degree $n$.

In the context of superconformal field theories, the notion of conformal vertex algebras of Definition 3 is generalized to superconformal vertex algebras. For an $N=2$ superconformal vertex algebra, the vectorspace $W$ in the above Definition is graded by $\frac{1}{2} \mathbb{Z}$ instead of $\mathbb{Z}$, one needs to allow odd fields $A(z)=Y(A, z)$, which can 
have mode expansions in $\left.z^{1 / 2} \cdot \operatorname{End}(W)\left[z^{ \pm 1}\right]\right]$, and one needs to generalize the notion of locality to such fields, as explained in the discussion of Definition 2 Finally, one needs to assume that there exist special states $J \in W_{1}$ and $G^{ \pm} \in W_{3 / 2}$ such that the associated fields $J(z), G^{ \pm}(z)$ obey the $N=2$ superconformal algebra (9)-10).

An important ingredient of CFT are the so-called $n$-point functions, which associate a function in $n$ complex variables to every $n$-tuple of states in the CFT. These $n$-point functions are naturally related to the notion of vertex algebras, as we shall illustrate now. Assume that $W$ is the $\mathbb{Z}$-graded vectorspace which underlies a conformal vertex algebra, with notations as in Definition 3 . Furthermore, assume that $W$ comes equipped with a positive definite scalar product $\langle\cdot, \cdot\rangle$, such that $W=\oplus_{m \in \mathbb{Z}} W_{m}$ is an orthogonal direct sum. Let $A(z), B(w)$ denote the fields associated to $A, B \in W$ by the state-field correspondence, which are local with respect to each other by the locality axiom. Hence by the very Definition 2 of locality, the formal power series $\langle\Omega, A(z) B(w) \Omega\rangle$ and $\langle\Omega, B(w) A(z) \Omega\rangle$ are obtained from the same series in $\mathbb{C}[[z, w]]\left[z^{-1}, w^{-1},(z-w)^{-1}\right]$ by means of the (formal) Taylor expansions $l_{z>w}$ and $l_{w>z}$, respectively. This series is denoted by $\langle A(z) B(w)\rangle \in$ $\mathbb{C}[[z, w]]\left[z^{-1}, w^{-1},(z-w)^{-1}\right]$, such that

$$
\boldsymbol{l}_{z>w}(\langle A(z) B(w)\rangle)=\langle\Omega, A(z) B(w) \Omega\rangle, \quad \boldsymbol{l}_{w>z}(\langle A(z) B(w)\rangle)=\langle\Omega, B(w) A(z) \Omega\rangle .
$$

Then $\langle A(z) B(w)\rangle$ is an example of a 2-point function, and for $A_{1}, \ldots, A_{n} \in W$ one analogously defines the $n$-point functions $\left\langle A_{1}\left(z_{1}\right) \cdots A_{n}\left(z_{n}\right)\right\rangle$ by successive OPE. The additional properties of CFTs ensure that these $n$-point functions define meromorphic functions in complex variables $z_{1}, \ldots, z_{n} \in \mathbb{C}$, whose possible poles are restricted to the partial diagonals $z_{i}=z_{j}, i \neq j$.

\subsection{Defining conformal field theories}

This section summarizes an axiomatic approach to conformal field theory. Instead of a full account, the focus lies on those ingredients of CFTs that are relevant for the remaining sections of this exposition. More details can be found e.g. in [Wen, Wen10]. We list the ingredients and defining properties of a two-dimensional Euclidean unitary conformal field theory at central charges $c, \bar{c}$ :

Ingredient I. [The SPACE OF STATES $\mathbb{H}$ ]

The space $\mathbb{H}$ is a $\mathbb{C}$-vectorspace with positive definite scalar product $\langle\cdot, \cdot\rangle$ and with a compatible real structure $v \mapsto v^{*}$. Furthermore, there are two Virasoro fields $T(z), \bar{T}(\bar{z})$ of central charges $c, \bar{c}$ on $\mathbb{H}$, see equation (1), where the OPE between $T$ and $\bar{T}$ is trivial:

$$
T(z) \bar{T}(\bar{z}) \sim 0
$$


The space of states of a CFT must have a number of additional properties:

Property A. The space of states $\mathbb{H}$ furnishes a UNITARY representation of the two commuting copies of a Virasoro algebra generated by the modes $L_{n}, \bar{L}_{n}, n \in \mathbb{Z}$, of the Virasoro fields $T(z)$ and $\bar{T}(\bar{z})$, which is COMPATIBLE WITH THE REAL STRUCTURE of $\mathbb{H}$. The central elements $c, \bar{c}$ act by multiplication with fixed, real constants, also denoted $c, \bar{c} \in \mathbb{R}$. The operators $L_{0}$ and $\bar{L}_{0}$ are self-adjoint and positive semidefinite, and $\mathbb{H}$ decomposes into a direct sum of their simultaneous eigenspaces indexed by $R \subset \mathbb{R}^{2}$,

$$
\mathbb{H}=\bigoplus_{(h, \bar{h}) \in R} \mathbb{H}_{h, \bar{h}}, \quad \mathbb{H}_{h, \bar{h}}:=\operatorname{ker}\left(L_{0}-h \cdot \mathrm{id}\right) \cap \operatorname{ker}\left(\bar{L}_{0}-\bar{h} \cdot \mathrm{id}\right)
$$

By this we mean that $R$ does not have accumulation points, and that every vector in $\mathbb{H}$ is a sum of contributions from finitely many different eigenspaces $\mathbb{H}_{h, \bar{h}}$. Moreover, every $\mathbb{H}_{h, \bar{h}}$ is finite dimensional.

Property $\mathrm{A}$ ensures that the space of states $\mathbb{H}$ of every conformal field theory furnishes a very well-behaved representation of two commuting copies of a Virasoro algebra. In addition, we need to assume that the CHARACTER of this representation has favorable properties:

Property B. For $\tau \in \mathbb{C}, \mathfrak{I}(\tau)>0$, let $q:=\exp (2 \pi i \tau)$; the PARTITION FUNCTION

$$
Z(\tau):=\sum_{(h, \bar{h}) \in R}\left(\operatorname{dim}_{\mathbb{C}} \mathbb{H}_{h, \bar{h}}\right) q^{h-c / 24} \bar{q}^{\bar{h}-\bar{c} / 24}=\operatorname{Tr}_{\mathbb{H}}\left(q^{L_{0}-c / 24} \bar{q}^{\bar{L}_{0}-\bar{c} / 24}\right)
$$

is well defined for all values of $\tau$ in the complex upper halfplane, and it is invariant under modular transformations

$$
\tau \mapsto \frac{a \tau+b}{c \tau+d}, \quad\left(\begin{array}{ll}
a & b \\
c & d
\end{array}\right) \in S L(2, \mathbb{Z}) .
$$

Since by Property $B$ the partition function is modular invariant, it in particular is invariant under the translation $\tau \mapsto \tau+1$ of the modular parameter. This implies that for every pair $(h, \bar{h}) \in R$ of eigenvalues of $L_{0}$ and $\bar{L}_{0}$, we have $h-\bar{h} \in \mathbb{Z}$. Hence the subspaces $W:=\operatorname{ker}\left(\bar{L}_{0}\right)$ and $\bar{W}:=\operatorname{ker}\left(L_{0}\right)$ are $\mathbb{Z}$-graded by $L_{0}$ and $\bar{L}_{0}$, respectively. To obtain a CFT, these subspaces are required to carry additional structure, which we are already familiar with:

Property C. The subspaces $W:=\operatorname{ker}\left(\bar{L}_{0}\right)$ and $\bar{W}:=\operatorname{ker}\left(L_{0}\right)$ of $\mathbb{H}$ carry the structure of conformal vertex algebras, see Definition 3 , with $T(z)$ and $\bar{T}(\bar{z})$ the fields associated to the respective conformal vectors by the state-field correspondence. Moreover, the vacuum vector $\Omega$ of the conformal vertex algebra 
$W$ agrees with the vacuum vector of $\bar{W}$, and $\Omega$ is a real unit vector yielding a basis of $W \cap \bar{W}=\mathbb{H}_{0,0}$.

The vertex algebras with underlying vectorspaces $W$ and $\bar{W}$ are called the CHIRAL ALGEBRAS of the CFT, and to simplify the terminology, we also refer to $W$ and $\bar{W}$ as the chiral algebras.

As was discussed at the end of Section 2.1 in this setting there is a natural definition of $n$-point functions for the fields in the chiral algebras associated to $W$ and $\bar{W}$. This definition, however, is not sufficient to capture the general $n$-point functions of conformal field theory. The notion is generalized along the following lines:

Ingredient II. [The system $\langle\cdots\rangle$ of $n$-point functions]

The space of states $\mathbb{H}$ is equipped with a system $\langle\cdots\rangle$ of $n$-POINT FUNCTIONS, that is, for every $n \in \mathbb{N}$ we have a map

$$
\mathbb{H}^{\otimes n} \longrightarrow \operatorname{Maps}\left(\mathbb{C}^{n} \backslash \bigcup_{i \neq j} D_{i, j}, \mathbb{C}\right), \quad D_{i, j}:=\left\{\left(z_{1}, \ldots, z_{n}\right) \in \mathbb{C}^{n} \mid z_{i}=z_{j}\right\},
$$

which is compatible with complex conjugation, and such that every function in the image is real analytic and allows an appropriate expansion about every partial diagonal $D_{i, j}$.

The following Property $\mathrm{D}$ which along with Property E governs the behavior of the $n$-point functions, is immediate on the chiral algebras $W$ and $\bar{W}$, by definition:

Property D. The $n$-point functions are LOCAL, that is, for every permutation $\sigma \in S_{n}$ and all $\phi_{i} \in \mathbb{H}$,

$$
\left\langle\phi_{1}\left(z_{1}\right) \cdots \phi_{n}\left(z_{n}\right)\right\rangle=\left\langle\phi_{\sigma(1)}\left(z_{\sigma(1)}\right) \cdots \phi_{\sigma(n)}\left(z_{\sigma(n)}\right)\right\rangle .
$$

Consider an $n$-point function $\left\langle\phi\left(z_{1}\right) \cdots \phi\left(z_{n}\right)\right\rangle$ with $\phi=\phi_{1}=\cdots=\phi_{n} \in \mathbb{H}$ as a function of one complex variable $z=z_{k}$, while all other $z_{l}, l \neq k$, are fixed. The closure of the domain of definition of this function is the WORLDSHEET on which the $n$-point function is defined. Therefore, Ingredient $\square$ yields $n$-point functions whose worldsheet is the Riemann sphere $\overline{\mathbb{C}}$. As a basic feature of conformal field theory, the $n$-point functions are assumed to transform covariantly under conformal maps between worldsheets. In particular,

Property E. The $n$-point functions are POINCARÉ COVARIANT, that is, for all isometries and all dilations $f$ of the Euclidean plane $\mathbb{C}$, and for all $\phi_{i} \in$ $\mathbb{H}_{h_{i}, \bar{h}_{i}}$,

$$
\left\langle\phi_{1}\left(f\left(z_{1}\right)\right) \cdots \phi_{n}\left(f\left(z_{n}\right)\right)\right\rangle=\prod_{i=1}^{n}\left[\left(f^{\prime}\left(z_{i}\right)\right)^{-h_{i}}\left(\overline{f^{\prime}\left(z_{i}\right)}\right)^{-\bar{h}_{i}}\right]\left\langle\phi_{1}\left(z_{1}\right) \cdots \phi_{n}\left(z_{n}\right)\right\rangle,
$$


where $f^{\prime}(z)=\partial_{z} f(z)$. Moreover, Infinitesimal TRANSLATIONS $\alpha L_{1}+$ $\bar{\alpha} \bar{L}_{1}, \alpha, \bar{\alpha} \in \mathbb{C}$, are represented by $\alpha \partial_{z}+\bar{\alpha} \partial_{\bar{z}}$, i.e. for arbitrary $\phi_{i} \in \mathbb{H}$,

$$
\begin{aligned}
& \left\langle\phi_{1}\left(z_{1}\right) \cdots \phi_{n-1}\left(z_{n-1}\right)\left(L_{1} \phi_{n}\right)\left(z_{n}\right)\right\rangle=\frac{\partial}{\partial z_{n}}\left\langle\phi_{1}\left(z_{1}\right) \cdots \phi_{n-1}\left(z_{n-1}\right) \phi_{n}\left(z_{n}\right)\right\rangle, \\
& \left\langle\phi_{1}\left(z_{1}\right) \cdots \phi_{n-1}\left(z_{n-1}\right)\left(\bar{L}_{1} \phi_{n}\right)\left(z_{n}\right)\right\rangle=\frac{\partial}{\partial \bar{z}_{n}}\left\langle\phi_{1}\left(z_{1}\right) \cdots \phi_{n-1}\left(z_{n-1}\right) \phi_{n}\left(z_{n}\right)\right\rangle .
\end{aligned}
$$

The remaining requirements on the $n$-point functions, unfortunately, are rather more involved. Roughly, they firstly generalize Property Eby ensuring that the representation of the two commuting copies of the Virasoro algebra on $\mathbb{H}$ (see Property A induces an action by infinitesimal conformal transformations on the worldsheet. Furthermore, the operator product expansion of Definition_2is generalized to induce the appropriate expansions of the $n$-point functions about partial diagonals, see Ingredient $\Pi$ Finally, $n$-point functions must be defined on worldsheets with arbitrary genus. Since these additional properties are not needed explicitly in the remaining sections of the present exposition, here only the relevant keywords are listed in the final

Property F. The system $\langle\cdots\rangle$ of $n$-point functions is CONFORMALLY COVARIANT, and it REPRESENTS AN OPERATOR PRODUCT EXPANSION such that REFLECTION POSITIVITY holds. Moreover, the UNIVERSALITY CONDITION holds, and sewing allows to define $n$-point functions on WORLDSHEETS OF ARBITRARY GENUS.

As was mentioned at the beginning of this section, the ingredients of CFTs listed above yield two-dimensional Euclidean unitary conformal field theories. Indeed, these adjectives have been implemented in Properties $\mathrm{A} F$ according to the discussion that precedes Property $\mathrm{E}$ along with Property $\mathrm{F}$ the worldsheets of our CFTs are two-dimensional Euclidean manifolds. Conformality is implemented by means of the two commuting copies of the Virasoro algebra, see the discussion of equations (1) and (2), which act by infinitesimal conformal transformations on the worldsheets of the $n$-point functions by Properties E and $\mathbb{F}$ On the space of states $\mathbb{H}$, Property A ensures that the representation of the infinitesimal conformal transformations is unitary.

Our approach to CFT is convenient, since it concretely implements the interplay between representation theory with the analytic properties of the $n$-point functions, which is characteristic of two-dimensional conformal quantum field theories. However, the relation to more general quantum field theories (QFTs) is not so evident. Let us briefly comment on this connection.

First, for the relevant QFTs we restrict to Euclidean quantum field theories according to a system of axioms that are based on the OSTERWALDER-SCHRADER AXIOMS [OS73, OS75], see [FFK89, Sch08]. According to [OS73, OS75], these 
axioms ensure that from such a QFT one can construct a Hilbert space $\widetilde{\mathbb{H}}$ of states $\phi$ and associated fields $Y_{\phi}$, where each field $Y_{\phi}$ yields a densely defined linear operator $Y_{\phi}(h)$ on $\widetilde{\mathbb{H}}$ for every test function $h$. Moreover, there is a special state $\Omega$ which plays the role of the vacuum as in our Property $\mathrm{C}$

The Osterwalder-Schrader axioms require the existence of correlation functions associated to every $n$-tuple of states in $\mathbb{H}$ which resemble the $n$-point functions of CFT according to our Ingredient $\Pi$ To obtain the fields of CFT from those of the general QFT, one needs to perform a procedure called LOCALIZATION. Within the Hilbert space $\widetilde{\mathbb{H}}$ one restricts to the subspace $\mathbb{H}$ which is generated by those states that are created by the localized field operators from the vacuum, generalizing the vacuum axiom of our Definition 3. The Osterwalder-Schrader axioms then ensure that locality (Property D), Poincaré covariance under isometries (Property E) and reflection positivity (Property $\mathrm{F}$ hold for the $n$-point functions obtained from the correlation functions of the QFT.

According to [FFK89], conformal covariance can be implemented by means of three additional axioms, ensuring the covariance of the $n$-point functions under di-

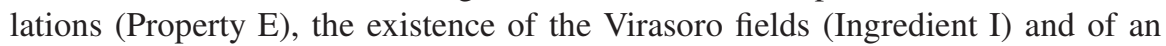
OPE (Properties C and F] with all the necessary features. See [Sch08, §9.3] for an excellent account.

If a CFT is obtained from a conformally covariant QFT by localization, then one often says that the CFT is the SHORT DISTANCE LIMIT of the QFT. For details on this mathematical procedure see [FFK89, FG94, Was95]. To the author's knowledge, it is unknown whether a CFT in the sense of our approach can always be viewed as a short distance limit of a full-fledged QFT.

With the above, we do not claim to provide a minimal axiomatic approach to CFT. For example, the requirement of Property $\mathbf{E}$ that $n$-point functions are welldefined on worldsheets of arbitrary genus implies modular invariance of the partition function, which was assumed separately in Property B. Indeed, the partition function $Z(\tau)$ is the 0 -point function on a worldsheet torus with modulus $\tau$, where conformal invariance implies that $Z(\tau)$ indeed solely depends on the complex structure represented by $\tau \in \mathbb{C}, \mathfrak{I}(\tau)>0$. Property $\mathbb{B}$ is stated separately for clarity, and because modular invariance plays a crucial role in the discussion of the elliptic genus in Section 2.4 which is also essential for the remaining sections of this exposition, while we refrain from a detailed discussion of Property $\mathrm{F}$

Mathematical implications of modular invariance for CFTs were first pointed out by Cardy [Car86. He observed that for those theories that had been studied by Belavin, Polyakov and Zamolodchikov in their seminal paper [BPZ84], and that describe physical phenomena in statistical physics, modular invariance of the partition function poses constraints on the operator content. These constraints can be useful for the classification of CFTs.

In special cases, modular invariance can be proven from first principles, assuming only that the $n$-point functions are well-defined on the Riemann sphere. In [Nah91], Nahm argues that the assumption that the $n$-point functions on the torus define thermal states of the field algebra, which in turn is of type I, suffices to deduce mod- 
ular invariance. Under an assumption known as CONDITION $C$ or CONDITION $C_{2}$, which amounts to certain quotients of the chiral algebras being finite dimensional, Zhu proves in [Zhu96] that modular invariance follows, as well. This covers a large class of examples of CFTs, among them the ones studied by Belavin, Polyakov and Zamolodchikov.

An $N=(2,2)$ SUPERCONFORMAL FIELD THEORY is a CFT as above, where the notion of locality is generalized according to what was said in the discussion of Definition 2] and the representations of the two commuting copies of a Virasoro algebra are extended to representations of $N=2$ superconformal algebras, see equations (9)-(10). As a first additional ingredient to these theories one therefore needs

Ingredient III. [Compatible $\mathbb{Z}_{2}$-grading of the space of states]

The space of states $\mathbb{H}$ carries a $\mathbb{Z}_{2}$-grading $\mathbb{H}=\mathbb{H}_{b} \oplus \mathbb{H}_{f}$ into WORLDSHEET BOSONS $\mathbb{H}_{b}$ (even) and WORLDSHEET FERMIONS $\mathbb{H}_{f}$ (odd), which is compatible with Properties $\mathrm{A} \mathrm{F}$

In more detail, for compatibility with Property $\mathrm{A}$ the decomposition $\mathbb{H}=$ $\mathbb{H}_{b} \oplus \mathbb{H}_{f}$ must be orthogonal and invariant under the action of the two commuting copies of the Virasoro algebra. In Property B, the trace defining the partition function is taken over the bosonic subspace $\mathbb{H}_{b}$, only. The chiral algebras introduced in Property $[$ must contain $N=2$ superconformal vertex algebras as introduced in the discussion of Definition 3 whose modes act unitarily on $\mathbb{H}$. The notion of locality in Property $\mathrm{D}$ is generalized to SEMILOCALITY, meaning that

$$
\left\langle\phi_{1}\left(z_{1}\right) \cdots \phi_{n}\left(z_{n}\right)\right\rangle=(-1)^{I}\left\langle\phi_{\sigma(1)}\left(z_{\sigma(1)}\right) \cdots \phi_{\sigma(n)}\left(z_{\sigma(n)}\right)\right\rangle
$$

if $\sigma \in S_{n}$ and all $\phi_{i} \in \mathbb{H}$ have definite parity. Here, $I$ is the number of inversions of odd states in $\sigma$, that is, the number of pairs $(i, j)$ of indices with $i<j$ and $\sigma(i)>\sigma(j)$ and such that $\phi_{i}, \phi_{j} \in \mathbb{H}_{f}$. Properties EandEremain unchanged.

The fields in the chiral algebras of the CFT that furnish the two commuting copies of $N=2$ superconformal vertex algebras according to Property $\amalg$ are generally denoted $T(z), J(z), G^{+}(z), G^{-}(z)$ and $\bar{T}(\bar{z}), \bar{J}(\bar{z}), \bar{G}^{+}(\bar{z}), \bar{G}^{-}(\bar{z})$ with OPEs as in (9)(10). The mode expansions for the even fields are denoted as

$$
T(z)=\sum_{n} L_{n} z^{n-2}, J(z)=\sum_{n} J_{n} z^{n-1}, \bar{T}(\bar{z})=\sum_{n} \bar{L}_{n} \bar{z}^{n-2}, \bar{J}(\bar{z})=\sum_{n} \bar{J}_{n} \bar{z}^{n-1},
$$

in accord with Definition 3 As mentioned in the discussion after Definition 3 the odd fields $G^{ \pm}(z)$ can have mode expansions either in $\operatorname{End}_{\mathbb{C}}(\mathbb{H})\left[\left[z^{ \pm 1}\right]\right]$ or in $z^{1 / 2}$. $\operatorname{End}_{\mathbb{C}}(\mathbb{H})\left[z^{ \pm 1}\right]$, and analogously for $\bar{G}^{ \pm}(\bar{z})$. This induces another $\mathbb{Z}_{2} \times \mathbb{Z}_{2}$ grading of the space of states $\mathbb{H}$,

$$
\mathbb{H}=\mathbb{H}^{N S, N S} \oplus \mathbb{H}^{R, R} \oplus \mathbb{H}^{N S, R} \oplus \mathbb{H}^{R, N S},
$$


where

$$
\left.G^{ \pm}(z) \in \operatorname{End}_{\mathbb{C}}\left(\mathbb{H}^{N S, \bullet}\right)\left[z^{ \pm 1}\right]\right], \quad \bar{G}^{ \pm}(\bar{z}) \in \operatorname{End}_{\mathbb{C}}\left(\mathbb{H}^{\bullet}, N S\right)\left[\left[\bar{z}^{ \pm 1}\right]\right]
$$

in the so-called NEVEU-SCHWARZ- or NS-SECTOR, while

$$
G^{ \pm}(z) \in z^{1 / 2} \cdot \operatorname{End}_{\mathbb{C}}\left(\mathbb{H}^{R, \bullet}\right)\left[\left[z^{ \pm 1}\right]\right], \quad \bar{G}^{ \pm}(\bar{z}) \in \bar{z}^{1 / 2} \cdot \operatorname{End}_{\mathbb{C}}\left(\mathbb{H}^{\bullet}, R\right)\left[\left[\bar{z}^{ \pm 1}\right]\right]
$$

in the so-called RAMOND- or R-SECTOR. That is, on $\mathbb{H}^{S, \bar{S}}$ the fields $G^{ \pm}(z)$ and $\bar{G}^{ \pm}(\bar{z})$ have mode expansions according to the $S$ and the $\bar{S}$ sector, respectively, with $S, \bar{S} \in\{R, N S\}$.

In what follows, we restrict our attention to so-called NON-CHIRAL $N=(2,2)$ SUPERCONFORMAL FIELD THEORIES WITH SPACE-TIME SUPERSYMMETRY:

\section{Ingredient IV. [Space-time supersymmetry]}

The space of states $\mathbb{H}$ carries another compatible $\mathbb{Z}_{2}$-grading by means of the properties of the odd fields $G^{ \pm}(z)$ and $\bar{G}^{ \pm}(\bar{z})$ of the $N=2$ superconformal vertex algebra into

$$
\mathbb{H}=\mathbb{H}^{N S} \oplus \mathbb{H}^{R} .
$$

Here, the decomposition (12) reduces to $\mathbb{H}^{N S}:=\mathbb{H}^{N S, N S}$ and $\mathbb{H}^{R}:=\mathbb{H}^{R, R}$, while the sectors $\mathbb{H}^{N S, R}$ and $\mathbb{H}^{R, N S}$ are trivial.

Moreover, as representations of the two commuting $N=2$ superconformal algebras of Ingredient $\llbracket \mathbb{H} \mathbb{H}^{N S}$ and $\mathbb{H}^{R}$ are related by an isomorphism $\Theta: \mathbb{H} \rightarrow \mathbb{H}$ which interchanges $\mathbb{H}^{N S}$ and $\mathbb{H}^{R}$ and which obeys

$$
\begin{array}{ll}
{\left[L_{0}, \Theta\right]=\frac{c}{24} \Theta-\frac{1}{2} \Theta \circ J_{0},} & {\left[J_{0}, \Theta\right]=-\frac{c}{6} \Theta,} \\
{\left[\bar{L}_{0}, \Theta\right]=\frac{\bar{c}}{24} \Theta-\frac{1}{2} \Theta \circ \bar{J}_{0},} & {\left[\bar{J}_{0}, \Theta\right]=-\frac{\bar{c}}{6} \Theta,}
\end{array}
$$

where $L_{0}, J_{0}, \bar{L}_{0}, \bar{J}_{0}$ are the zero-modes of the fields $T(z), J(z), \bar{T}(\bar{z}), \bar{J}(\bar{z})$ obtained from the mode expansions 111). The isomorphism $\Theta$ is induced by a field of the theory called SPECTRAL FLOW, and it is also known as SPACETIME SUPERSYMMETRY.

The spectral flow induces an inner automorphism $X \mapsto X^{\prime}=\Theta X \Theta^{-1}$ on the $N=$ $(2,2)$ superconformal algebra, with the following beautiful property: our standard generators $\left(X_{n}\right)_{X \in\left\{L, J, G^{ \pm}\right\}, n \in \mathbb{Z}}$ in the Ramond sector are mapped to generators $X_{r}^{\prime}$ in the Neveu-Schwarz sector yielding the modes of fields $T^{\prime}, J^{\prime}, G^{ \pm \prime}$ that obey the $N=$ 2 superconformal algebra (9)-(10), and analogously for the right-moving fields. As such, spectral flow implements an equivalence of representations of the $N=(2,2)$ superconformal algebra.

With the above notion of CFT, a number of examples are known, like minimal models, both the bosonic [BPZ84, GKO86] and the supersymmetric ones [DPYZ86, BFK86, ZF86, Qiu87]. In string theory, so-called non-linear sigma model constructions are believed to provide a map from certain manifolds to CFTs. While this construction is well understood for the simplest manifolds, namely for tori, the mathematical details in general are far from understood. 


\subsection{Example: toroidal conformal field theories}

For illustration and for later reference, this section very briefly presents the class of so-called TOROIDAL CONFORMAL FIELD THEORIES. These theories are characterized by the existence of "sufficiently many $U(1)$-currents" as in Example 1 of Section 2.1.

We say that the chiral algebra $W=\oplus_{m} W_{m}$ of a CFT (see Property C. Section 2.2 CONTAINS A $\mathfrak{u}(1)^{d}$-CURRENT ALGEBRA, if $W_{1}$ contains an orthogonal system $\left(a_{1}^{k} \Omega, k \in\{1, \ldots, d\}\right)$ of states, which under the state-field correspondence of Definition 3 have associated fields $j^{k}(z), k \in\{1, \ldots, d\}$, obeying the OPEs

$$
\forall k, l \in\{1, \ldots, d\}: \quad j^{k}(z) j^{l}(w) \sim \frac{\delta^{k, l}}{(z-w)^{2}} .
$$

For (bosonic) CFTs we then have

Definition 4. A conformal field theory at central charges $c, \bar{c}$ is called TOROIDAL, if $c=\bar{c}=d$ with $d \in \mathbb{N}$, and if the chiral algebras $W=\oplus_{m} W_{m}$, $\bar{W}=\oplus_{m} \bar{W}_{m}$ of PropertyC Section 2.2, each contain a $\mathfrak{u}(1)^{d}$-current algebra.

For our purposes, the TOROIDAL $N=(2,2)$ SUPERCONFORMAL FIELD THEORIES are more relevant. They are characterized by the fact that their bosonic sector with space of states $\mathbb{H}_{b}$ contains a toroidal CFT at central charges $2 D, 2 D$ in the sense of Definition 4 , and in addition, they contain $D$ left- and $D$ right-moving socalled DIRAC FERMIONS WITH COUPLED SPIN STRUCTURES. By this we mean first of all that the subspace $W_{1 / 2} \subset W$ of the vectorspace underlying the chiral algebra contains an orthogonal system $\left(\left(\psi_{ \pm}^{k}\right)_{1 / 2} \Omega, k \in\{1, \ldots, D\}\right)$ of states, which under the state-field correspondence of Definition 3 have associated (odd) fields $\psi_{k}^{ \pm}(z), k \in\{1, \ldots, D\}$, obeying the OPEs

$$
\forall k, l \in\{1, \ldots, D\}: \quad \psi_{k}^{+}(z) \psi_{l}^{-}(w) \sim \frac{\delta^{k, l}}{z-w}, \quad \psi_{k}^{ \pm}(z) \psi_{l}^{ \pm}(w) \sim 0,
$$

and analogously for the subspace $\bar{W}_{1 / 2} \subset \bar{W}$ of the vectorspace underlying the second chiral algebra in Property $\left[\right.$. In addition, all $\psi_{k}^{ \pm}(z)$ are represented by formal power series in $\operatorname{End}_{\mathbb{C}}\left(\mathbb{H}^{N S}\right)\left[\left[z^{ \pm 1}\right]\right]$ on $\mathbb{H}^{N S}$, while on $\mathbb{H}^{R}$, they are represented in $z^{1 / 2} \cdot \operatorname{End}_{\mathbb{C}}\left(\mathbb{H}^{R}\right)\left[\left[z^{ \pm 1}\right]\right]$, and analogously for the $\bar{\psi}_{k}^{ \pm}(\bar{z})$. One shows that such a system of $D$ left- and $D$ right-moving Dirac fermions yields a well-defined CFT at central charges $D, D$ (see e.g. [Gin88, §8.2]).

Definition 5. An $N=(2,2)$ superconformal field theory at central charges $c, \bar{c}$ with space-time supersymmetry is toroidal, if $c=\bar{c}=3 D$ with $D \in \mathbb{N}$, and if this theory is the tensor product of a toroidal conformal field theory 
at central charges $2 D, 2 D$ according to Definition 4 and a system of $D$ leftand $D$ right-moving Dirac fermions with coupled spin structures. Moreover, the fields $\psi_{k}^{ \pm}(z), k \in\{1, \ldots, D\}$, in (15) yield the superpartners of the $U(1)$ currents $j^{l}(z), l \in\{1, \ldots, 2 D\}$, in (14), and analogously for the right-moving fields. By this we mean that for the fields $G^{ \pm}(z), \bar{G}^{ \pm}(\bar{z})$ in the two commuting superconformal vertex algebras (9)-(10), and with notations as above, we have

$$
\begin{aligned}
\forall k \in\{1, \ldots, D\}: & G_{-1 / 2}^{ \pm} a_{1}^{k} \Omega=\left(\psi_{ \pm}^{k}\right)_{1 / 2} \Omega, G_{-1 / 2}^{ \pm} a_{1}^{k+D} \Omega=\mp i\left(\psi_{ \pm}^{k}\right)_{1 / 2} \Omega, \\
\bar{G}_{-1 / 2}^{ \pm} \bar{a}_{1}^{k} \Omega & =\left(\bar{\psi}_{ \pm}^{k}\right)_{1 / 2} \Omega, \bar{G}_{-1 / 2}^{ \pm} \bar{a}_{1}^{k+D} \Omega=\mp i\left(\bar{\psi}_{ \pm}^{k}\right)_{1 / 2} \Omega .
\end{aligned}
$$

The toroidal conformal and superconformal field theories have been very well understood by string theorists since the mid eighties [CENT85, Nar86], and these theories have also been reformulated in terms of the vertex algebras presented in Section 2.1] [Kac98, KO03, FBZ04]. This includes the interpretation of the toroidal conformal field theories as non-linear sigma models on tori, their deformations, and thus the structure of the moduli space of toroidal CFTs:

Theorem 1 ([Nar86]). The moduli space $\mathscr{M}_{D}^{\text {tor }}$ of toroidal $N=(2,2)$ superconformal field theories at central charges $c=\bar{c}=3 D$ with $D \in \mathbb{N}$ is a quotient of a $4 D^{2}$-dimensional Grassmannian by an infinite discrete group,

$$
\begin{aligned}
\mathscr{M}_{D}^{\text {tor }}=O^{+}(2 D, 2 D ; \mathbb{Z}) \backslash \mathscr{T}^{2 D, 2 D}, \\
\text { where } \mathscr{T}^{2 D, 2 D}:=O^{+}(2 D, 2 D ; \mathbb{R}) / S O(2 D) \times O(2 D) .
\end{aligned}
$$

Here, if $p q \neq 0$, then $O^{+}(p, q ; \mathbb{R})$ denotes the group of those elements in $O(p, q ; \mathbb{R})=O\left(\mathbb{R}^{p, q}\right)$ which preserve the orientation of maximal positive definite oriented subspaces in $\mathbb{R}^{p, q}$, and if $p \equiv q \bmod 8$, then $O^{+}(p, q ; \mathbb{Z})=$ $O^{+}(p, q ; \mathbb{R}) \cap O\left(\mathbb{Z}^{p, q}\right)$ with $\mathbb{Z}^{p, q} \subset \mathbb{R}^{p, q}$ the standard even unimodular lattice of signature $(p, q)$.

\subsection{The elliptic genus}

In this section, the conformal field theoretic elliptic genus is introduced and compared to the complex elliptic genus that is known to topologists and geometers. This and the following section are completely expository with more details and proofs to be found in the literature as referenced.

Let us first consider an $N=(2,2)$ superconformal field theory at central charges $c, \bar{c}$ with space-time supersymmetry according to the Ingredients IIV of Section 2.2. For the zero-modes $J_{0}, \bar{J}_{0}$ of the fields $J(z), \bar{J}(\bar{z})$ in the two commuting $N=2$ 
superconformal vertex algebras $T(z), J(z), G^{+}(z), G^{-}(z), \bar{T}(\bar{z}), \bar{J}(\bar{z}), \bar{G}^{+}(\bar{z}), \bar{G}^{-}(\bar{z})$ of Ingredient [11] according to (111) one finds: these linear operators are self-adjoint and simultaneously diagonalizable on the space of states $\mathbb{H}=\mathbb{H}^{N S} \oplus \mathbb{H}^{R}$. By Ingredient IV] the corresponding operator of spectral flow induces an equivalence of representations $\mathbb{H}^{N S} \cong \mathbb{H}^{R}$ of two $N=(2,2)$ superconformal algebras. This turns out to imply that the linear operator $J_{0}-\bar{J}_{0}$ has only integral eigenvalues, which are even on $\mathbb{H}_{b}$ and odd on $\mathbb{H}_{f}$, see e.g. [Wen00, §3.1]. Hence $(-1)^{J_{0}-\bar{J}_{0}}$ is an involution which yields the $\mathbb{Z}_{2}$-grading $\mathbb{H}=\mathbb{H}_{b} \oplus \mathbb{H}_{f}$ and an induced $\mathbb{Z}_{2}$-grading on $\mathbb{H}^{R}$. Following [EOTY89], this allows the definition of a supercharacter of the superconformal field theory, analogous to the partition function in Property B.

Definition 6. Consider an $N=(2,2)$ superconformal field theory at central charges $c, \bar{c}$ with space-time supersymmetry. Set $q:=\exp (2 \pi i \tau)$ for $\tau \in \mathbb{C}, \mathfrak{I}(\tau)>0$, and $y:=\exp (2 \pi i z)$ for $z \in \mathbb{C}$. Then

$$
\begin{aligned}
\mathscr{E}(\tau, z) & :=\operatorname{Str}_{\mathbb{H}^{R}}\left(y^{J_{0}} q^{L_{0}-c / 24} \bar{q}^{L_{0}-\bar{c} / 24}\right) \\
& =\operatorname{Tr}_{\mathbb{H}^{R}}\left((-1)^{J_{0}-\bar{J}_{0}} y^{J_{0}} q^{L_{0}-c / 24} \bar{q}^{L_{0}-\bar{c} / 24}\right)
\end{aligned}
$$

is the CONFORMAL FIELD THEORETIC ELLIPTIC GENUS of the theory.

Using known properties of the $N=2$ superconformal algebra and of its irreducible unitary representations, one shows (see [AKMW87, EOTY89, DY93, Wit94] for the original results and e.g. [Wen00, §3.1] for a summary and proofs):

Proposition 1. Consider the conformal field theoretic elliptic genus $\mathscr{E}(\tau, z)$ of an $N=(2,2)$ superconformal field theory at central charges $c, \bar{c}$ with spacetime supersymmetry.

Then $\mathscr{E}(\tau, z)$ is holomorphic in $\tau$ and bounded when $\tau \rightarrow i \infty$.

It is invariant under smooth deformations of the underlying superconformal field theory to any other space-time supersymmetric $N=(2,2)$ superconformal field theory with the same central charges.

Moreover, $\mathscr{E}(\tau, z)$ transforms covariantly under modular transformations,

$$
\mathscr{E}(\tau+1, z)=\mathscr{E}(\tau, z), \quad \mathscr{E}(-1 / \tau, z / \tau)=e^{2 \pi i \frac{c}{6} \cdot \frac{z^{2}}{\tau}} \mathscr{E}(\tau, z) .
$$

If in addition $c=\bar{c} \in 3 \mathbb{N}$, and all eigenvalues of $J_{0}$ and $\bar{J}_{0}$ in the Ramond sector lie in $\frac{c}{6}+\mathbb{Z}$, then

$$
\mathscr{E}(\tau, z+1)=(-1)^{\frac{c}{3}} \mathscr{E}(\tau, z), \quad \mathscr{E}(\tau, z+\tau)=q^{-\frac{c}{6}} y^{-\frac{c}{3}} \mathscr{E}(\tau, z) .
$$

In other words, $\mathscr{E}(\tau, z)$ is a WEAK JACOBI FORM (with a character, if $c / 3$ is odd) of WEIGHT 0 and INDEX $c / 6$. 
Note that the additional assumptions on the central charges and the eigenvalues of $J_{0}$ and $\bar{J}_{0}$ in the last statement of Proposition 1 are expected to hold for superconformal field theories that are obtained by a non-linear sigma model construction from some Calabi-Yau D-manifold [EOTY89].

On the other hand, following Hirzebruch's seminal work on multiplicative sequences and their genera [Hir66], the elliptic genus is known to topologists as a ring homomorphism from the cobordism ring of smooth oriented compact manifolds into a ring of modular functions [Lan88, Hir88]. For simplicity we assume that our underlying manifold $X$ is a Calabi-Yau $D$-manifold. Then its associated complex elliptic genus $\mathscr{E}_{X}(\tau, z)$ can be viewed as a modular function obeying the transformation properties of Proposition 1 with $c=3 D$ and interpolating between the standard topological invariants of $X$, namely its EULER CHARACTERISTIC $\chi(X)$, its SIGNATURE $\sigma(X)$, and its HOLOMORPHIC EULER CHARACTERISTIC $\chi\left(\mathscr{O}_{X}\right)$.

To understand this in more detail, first recall the definition of the topological invariants mentioned above: for $y \in \mathbb{C}$ the Hirzebruch $\chi_{y}$-genus [Hir66] is defined by

$$
\chi_{y}(X):=\sum_{p, q=0}^{D}(-1)^{q} y^{p} h^{p, q}(X),
$$

where the $h^{p, q}(X)$ are the Hodge numbers of $X$. Then

$$
\chi(X):=\chi_{-1}(X), \quad \sigma(X):=\chi_{+1}(X), \quad \chi\left(\mathscr{O}_{X}\right):=\chi_{0}(X) .
$$

Note that by the usual symmetries among the Hodge numbers $h^{p, q}(X)$ of a complex Kähler manifold $X$, the signature $\sigma(X)=\sum_{p, q}(-1)^{q} h^{p, q}(X)$ vanishes if the complex dimension $D$ of $X$ is odd; we have thus trivially extended the usual definition of the signature on oriented compact manifolds whose real dimension is divisible by 4 to all compact complex Kähler manifolds.

To motivate a standard formula for the specific elliptic genus which is of relevance to us, see Definition 7, we draw the analogy to the interpretation of the topological invariants (16) in terms of the Atiyah-Singer Index Theorem [AS63]. For any complex vector bundle $E$ on $X$ and a formal variable $x$, we introduce the shorthand notations

$$
\Lambda_{x} E:=\bigoplus_{p} x^{p} \Lambda^{p} E, \quad S_{x} E:=\bigoplus_{p} x^{p} S^{p} E,
$$

where $\Lambda^{p} E, S^{p} E$ denote the exterior and the symmetric powers of $E$, respectively, along with the CHERN CHARACTER on such formal power series in $x$ whose coefficients are complex vector bundles $F_{p}$ :

$$
\operatorname{ch}\left(\bigoplus_{p} x^{p} F_{p}\right):=\sum_{p} x^{p} \operatorname{ch}\left(F_{p}\right) .
$$


Then by the Hirzebruch-Riemann-Roch formula [Hir54], which can be viewed as a special case of the Atiyah-Singer Index Theorem, one finds

$$
\chi_{y}(X)=\int_{X} \operatorname{Td}(X) \operatorname{ch}\left(\Lambda_{y} T^{*}\right),
$$

where $\operatorname{Td}(X)$ denotes the TODD GENUS and $T:=T^{1,0} X$ is the holomorphic tangent bundle of $X$. Generalizing the expression in equation (17) and following [Hir88, Wit88a, Kri90] we now have

Definition 7. Let $X$ denote a compact complex $D$-manifold with holomorphic tangent bundle $T:=T^{1,0} X$. Set

$$
\mathbb{E}_{q,-y}:=y^{-D / 2} \bigotimes_{n=1}^{\infty}\left(\Lambda_{-y q^{n-1}} T^{*} \otimes \Lambda_{-y^{-1} q^{n}} T \otimes S_{q^{n}} T^{*} \otimes S_{q^{n}} T\right),
$$

viewed as a formal power series with variables $y^{ \pm 1 / 2}, q$, whose coefficients are holomorphic vector bundles on $X$.

Analogously to Definition 1 , the integral $\int_{X}$ is extended linearly to the vectorspace of formal power series whose coefficients are characteristic classes on $X$. Then with $q:=\exp (2 \pi i \tau)$ and $y:=\exp (2 \pi i z)$, the holomorphic Euler characteristic of $\mathbb{E}_{q,-y}$,

$$
\mathscr{E}_{X}(\tau, z):=\int_{X} \operatorname{Td}(X) \operatorname{ch}\left(\mathbb{E}_{q,-y}\right) \quad \in y^{-D / 2} \cdot \mathbb{Z}\left[\left[y^{ \pm 1}, q\right],\right.
$$

is the (complex) ELLIPTIC GENUS of $X$.

By [Hir88, Wit88a, Kri90], the elliptic genus $\mathscr{E}_{X}(\tau, z)$ in fact yields a well-defined function in $\tau \in \mathbb{C}$ with $\mathfrak{I}(\tau)>0$ and in $z \in \mathbb{C}$. If $X$ is a Calabi-Yau $D$-manifold, then $\mathscr{E}_{X}(\tau, z)$ is a weak Jacobi form (with a character, if $D$ is odd) of weight 0 and index $D / 2$ [BL00]. In other words, with $c:=3 D$ the elliptic genus $\mathscr{E}_{X}(\tau, z)$ obeys the transformation properties stated for $\mathscr{E}(\tau, z)$ in Proposition 11, and it is bounded when $\tau \rightarrow i \infty$. One checks that by definition, the elliptic genus indeed is a topological invariant which interpolates between the standard topological invariants of equation (16), namely $\mathscr{E}_{X}(\tau, z) \stackrel{\tau \rightarrow i \infty}{\longrightarrow} y^{-D / 2} \chi_{-y}(X)$ and

$$
\begin{aligned}
\mathscr{E}_{X}(\tau, z=0)=\chi(X), \quad \mathscr{E}_{X}(\tau, z=1 / 2) & =(-1)^{D / 2} \sigma(X)+\mathscr{O}(q), \\
q^{D / 4} \mathscr{E}_{X}(\tau, z=(\tau+1) / 2) & =(-1)^{D / 2} \chi\left(\mathscr{O}_{X}\right)+\mathscr{O}(q) .
\end{aligned}
$$

According to Witten Wit87. Wit88a], the expression for the elliptic genus $\mathscr{E}_{X}(\tau, z)$ in Definition 7 can be interpreted as a regularized version of a $U(1)$-equivariant index of a Dirac-like operator on the loop space of $X$, see also [Lan88]. This explains the notation chosen in Definition 7 and it also motivates why one expects that for CFTs which are obtained by a non-linear sigma model construction from 
some Calabi-Yau $D$-manifold $X$, the conformal field theoretic elliptic genus of Definition 6 agrees with the complex elliptic genus of $X$ as in Definition 7 Note that the resulting equation

$$
\mathscr{E}_{X}(\tau, z)=\operatorname{Str}_{\mathbb{H}^{R}}\left(y^{J_{0}} q^{L_{0}-c / 24} \bar{q}^{\bar{L}_{0}-\bar{c} / 24}\right)
$$

would furnish a natural generalization of the MCKEAN-SINGER FORMULA [jMS67]. While non-linear sigma model constructions are not understood sufficiently well to even attempt a general proof of this equation, there is some evidence for its truth. On the one hand, as was pointed out in Section 2.3. $N=(2,2)$ superconformal field theories obtained from a non-linear sigma model on a complex torus are very well understood. One confirms that their conformal field theoretic elliptic genus vanishes, as does the complex elliptic genus of a complex torus. Equation (19) is also compatible with the construction of symmetric powers of the manifold $X$ [DMVV97]. Moreover, compatibility of the elliptic genus with orbifold constructions was proved in [BL03, FS07]. Further evidence in favor of the expectation [19] arises from a discussion of the chiral de Rham complex, see Section 2.5

\subsection{The chiral de Rham complex}

As was pointed out above, non-linear sigma model constructions of $N=(2,2)$ superconformal field theories are in general not very well understood. Therefore, a direct proof of the expected equality 190 is out of reach. However, instead of a fullfledged superconformal field theory, in [MSV99] the authors construct a sheaf of superconformal vertex algebras, known as the CHIRAL DE RHAM COMPLEX $\Omega_{X}^{\text {ch }}$, on any complex manifold $X$. The chiral de Rham complex of $X$ is expected to be closely related to the non-linear sigma model on $X$, as we shall discuss in the present section.

Let us begin by summarizing the construction of the chiral de Rham complex $\Omega_{X}^{\text {ch }}$ for a complex $D$-dimensional manifold $X$, see [MSV99, GM04, LL07, BHS08]. First, to any coordinate neighborhood $U \subset X$ with holomorphic coordinates $\left(z^{1}, \ldots, z^{D}\right)$ one associates a $b c-\beta \gamma$ system $\Omega_{X}^{\text {ch }}(U)$ as in Example 3, see Section 2.1. Here, the even fields $a^{j}, b^{j}$ are interpreted as arising from quantizing the local sections $\partial / \partial z^{j}, z^{j}$ of the sheaf of polyvector fields on $X$, while the odd fields $\phi^{j}, \psi^{j}$ correspond to the local sections $d z^{j}, \partial / \partial\left(d z^{j}\right)$ of the sheaf of differential operators on the de Rham algebra of differential forms. Indeed, by (3) the map

$$
\left(\partial / \partial z^{j}, z^{j}, d z^{j}, \partial / \partial\left(d z^{j}\right)\right) \longmapsto\left(a_{0}^{j}, b_{0}^{j}, \phi_{0}^{j}, \psi_{0}^{j}\right)
$$

induces a super-Lie algebra homomorphism.

According to [MSV99], coordinate transforms on $X$ induce corresponding transformation rules for the fields $a^{j}, b^{j}, \phi^{j}, \psi^{j}$ which are compatible with the structure 
of the $b c-\beta \gamma$-system as discussed in Example 3 This allows to glue the $\Omega_{X}^{\text {ch }}(U)$ accordingly, and by localization, one indeed obtains a well-defined sheaf of vertex algebras over $X$, with a (non-associative) action of $\mathscr{O}_{X}$ on it.

A key result of [MSV99] is the fact that under appropriate assumptions on $X$, there are well-defined global sections of the sheaf $\operatorname{End}_{\mathbb{C}}\left(\Omega_{X}^{\mathrm{ch}}\right)\left[\left[z^{ \pm 1}\right]\right]$, which are locally given by the fields (4), (5) of the topological $N=2$ superconformal algebra (6)-(7) discussed in Example4 of Section 2.1.

Theorem 2 (|MSV99|). Let $X$ denote a compact complex manifold of dimension D. As discussed above, there is an associated sheaf $\Omega_{X}^{\mathrm{ch}}$ of vertex algebras on $X$. On every holomorphic coordinate chart $U \subset X$, let $T^{\mathrm{top}}(z), J(z), Q(z), G(z)$ denote the local sections in $\left.\operatorname{End}_{\mathbb{C}}\left(\Omega_{X}^{\mathrm{ch}}(U)\right)\left[z^{ \pm 1}\right]\right] d e-$ fined by (4), (5), with mode expansions

$$
T^{\mathrm{top}}(z)=\sum_{n} L_{n}^{\mathrm{top}} z^{n-2}, J(z)=\sum_{n} J_{n} z^{n-1}, Q(z)=\sum_{n} Q_{n} z^{n-1}, G(z)=\sum_{n} G_{n} z^{n-2},
$$

respectively. Then the following holds:

1. The linear operators $F:=J_{0}$ and $d_{\mathrm{dR}}^{\mathrm{ch}}:=-Q_{0}$ are globally well-defined. Moreover, $F$ defines a $\mathbb{Z}$-grading on $\Omega_{X}^{\mathrm{ch}}$, while $\left(d_{\mathrm{dR}}^{\text {ch }}\right)^{2}=0$, such that

$$
\forall p \in \mathbb{Z}: \quad \Omega_{X}^{\mathrm{ch}, p}(U):=\left\{\Phi \in \Omega_{X}^{\mathrm{ch}}(U) \mid F \Phi=p \Phi\right\}
$$

yields a complex $\left(\Omega_{X}^{\mathrm{ch}, \bullet}, d_{\mathrm{dR}}^{\mathrm{ch}}\right)$, which is called the CHIRAL DE RHAM COMPLEX.

2. The map $\left(z^{j}, d z^{j}\right) \mapsto\left(b_{0}^{j}, \phi_{0}^{j}\right)$ induces a quasi-isomorphism from the usual de Rham complex to the chiral de Rham complex of $X$.

3. The fields $T^{\text {top }}(z)$ given locally in (4) define a global field on the chiral de Rham complex, by which we mean a global section of the sheaf $\left.\operatorname{End}_{\mathbb{C}}\left(\Omega_{X}^{\mathrm{ch}}\right)\left[z^{ \pm 1}\right]\right]$. The chiral de Rham complex therefore is bigraded by $F$ and $L_{0}^{\text {top }}$.

4. If $X$ is a Calabi-Yau manifold, then the fields $J(z), Q(z), G(z)$ given locally in (5) also define global fields on the chiral de Rham complex.

As mentioned above, the sheaf $\Omega_{X}^{\mathrm{ch}}$ is not quasi-coherent. However, it has a filtration which is compatible with the bigrading of Theorem 2 and such that the corresponding graded object yields a quasi-coherent sheaf isomorphic to (the sheaf of sections of) $(-y)^{D / 2} \mathbb{E}_{q, y}$ as in Definition 7 This is used extensively in [Bor01, BL00] to study the Čech resolution of the sheaf cohomology $H^{*}\left(X, \Omega_{X}^{\text {ch }}\right)$. Note that this means classical Cech cohomology, ignoring the differential $d_{\mathrm{dR}}^{\mathrm{ch}}$ of the chiral de Rham complex. The authors of [Bor01, BL00] find: 
Theorem 3 ([Bor01, BL00|). Consider a Calabi-Yau D-manifold X, and the sheaf cohomology $H^{*}\left(X, \Omega_{X}^{\text {ch }}\right)$ of its chiral de Rham complex $\Omega_{X}^{\text {ch }}$. Equip it with the induced bigrading by the operators $F=J_{0}$ and $L_{0}^{\text {top }}$ of Theorem 2 and the $\mathbb{Z}_{2}$-grading by $(-1)^{F+q}$ on $H^{q}\left(X, \Omega_{X}^{c h}\right)$. Then $H^{*}\left(X, \Omega_{X}^{c h}\right)$ carries a natural structure of a superconformal vertex algebra, containing a topological $N=2$ superconformal algebra [Bor01, Prop. 3.7 and Def. 4.1]. Moreover [BL00], the GRADED EULER CHARACTERISTIC of the chiral de Rham complex, that is, the supertrace of the operator $y^{-D / 2} \cdot\left(y^{J_{0}} q^{L_{0}^{\text {top }}}\right)$ on $H^{*}\left(X, \Omega_{X}^{\text {ch }}\right)$, yields the elliptic genus $\mathscr{E}_{X}(\tau, z)$ of Definition 7

Thus Theorem 3 indicates a possible relationship between the chiral de Rham complex $\Omega_{X}^{\text {ch }}$ of a Calabi-Yau $D$-manifold $X$ and a non-linear sigma model on $X$, since it recovers the (geometrically defined!) elliptic genus $\mathscr{E}_{X}(\tau, z)$ by means of a supertrace which at least in spirit agrees with the expression on the right hand side of equation (19). Note that $L_{0}^{\text {top }}=L_{0}-\frac{1}{2} J_{0}$ by (8) (using Definition 11 and (11)). Therefore, using the fact that the elliptic genus is holomorphic, along with the spectral flow (13), the conformal field theoretic elliptic genus of Definition 6 can be expressed as

$$
\begin{aligned}
\mathscr{E}(\tau, z) & =\operatorname{Str}_{\mathbb{H}^{R}}\left(y^{J_{0}} q^{L_{0}-c / 24}\right) \\
& =y^{-c / 6} \operatorname{Str}_{\mathbb{H}^{N S}}\left(\left(y q^{-1 / 2}\right)^{J_{0}} q^{L_{0}}\right)=y^{-c / 6} \operatorname{Str}_{\mathbb{H}^{N S}}\left(y^{J_{0}} q^{L_{0}^{\mathrm{top}}}\right) .
\end{aligned}
$$

Hence recalling $c=3 D$ for a non-linear sigma model on a Calabi-Yau $D$-manifold, one is led to conjecture that one might be able to identify an appropriate cohomology of $\mathbb{H}^{N S}$ with $H^{*}\left(X, \Omega_{X}^{c h}\right)$.

The details of such an identification are still more subtle, however. Indeed, by construction, the chiral de Rham complex depends only on the complex structure of $X$, while the non-linear sigma model, in addition, depends on the complexified Kähler structure of $X$. It is therefore natural to expect the vertex algebra of Theorem 3 to yield a truncated version of the non-linear sigma model by means of the topological twists mentioned in Example 5 of Section 2.1. Since the crucial bundle $\mathbb{E}_{q,-y}$ of Definition 7 resembles an infinite-dimensional Fock space, while the traditional topological A- and B-twists yield finite dimensional spaces of states, the so-called HALF-TWISTED SIGMA MODEL according to Witten [Wit92] is the most natural candidate. It still cannot yield the vertex algebra of Theorem 3 , since it depends both on the complex and on the complexified Kähler structure of $X$. Moreover, the Čech resolution, which is implicit in $H^{*}\left(X, \Omega_{X}^{c h}\right)$, does not resemble the standard features of non-linear sigma models on $X$. According to Kapustin, however, an infinite volume limit of Witten's half twisted sigma model on $X$ yields the cohomology of $\Omega_{X}^{\text {ch }}$ by means of yet another resolution of the complex, the so-called DOLBEAULT RESOLUTION [Kap05]. 


\section{Conformal field theory on K3}

As emphasized repeatedly, non-linear sigma model constructions are in general not well understood, except for the toroidal conformal field theories presented in Section 2.3 Recall however that there are only two topologically distinct types of Calabi-Yau 2-manifolds, namely the complex 2-tori and the K3 surfaces (see e.g. [BHPvdV04, Ch.VIII] for an excellent introduction to the geometry of K3 surfaces). By the Kummer construction, one obtains an example of a K3 surface by means of a $\mathbb{Z}_{2}$-orbifold procedure from every complex 2 -torus. On the other hand, $\mathbb{Z}_{2 \text { - }}$ orbifolds of the toroidal CFTs are also reasonably well understood. One therefore expects to be able to construct examples of CFTs which allow a non-linear sigma model interpretation on some K3 surface. Compared to CFTs on higher-dimensional Calabi-Yau D-manifolds, those on K3 surfaces indeed provide a borderline case, in the sense that much more is known about these so-called K3 THEORIES. Most importantly, we can give a mathematical definition of such theories without ever mentioning non-linear sigma model constructions. The current section presents this definition and summarizes some of the known properties of K3 theories.

To motivate the mathematical definition of $\mathrm{K} 3$ theories, let us recall the conformal field theoretic elliptic genus of Section 2.4 Here we assume that we are given an $N=(2,2)$ superconformal field theory that obeys the following conditions, which are necessary for the CFT to allow a non-linear sigma model interpretation on some Calabi-Yau 2-manifold: the theory is superconformal at central charges $c=6, \bar{c}=6$ with space-time supersymmetry, and such that all eigenvalues of $J_{0}$ and $\bar{J}_{0}$ are integral. This latter condition is equivalent to the assumption that in addition to the spectral flow operator of Ingredient $\llbracket$ in Section 2.2, the theory possesses a quartet of TWO-FOLD LEFT- AND RIGHT-HANDED SPECTRAL FLOW OPERATORS $\Theta^{ \pm}, \bar{\Theta}^{ \pm}$. By this we mean that these operators act analogously to $\Theta^{ \pm 2}$ on the space of states, with $\Theta$ as in 13 , namely

$$
\begin{array}{ll}
{\left[L_{0}, \Theta^{ \pm}\right]=\frac{c}{6} \Theta^{ \pm} \mp \Theta^{ \pm} \circ J_{0},} & {\left[J_{0}, \Theta^{ \pm}\right]=\mp \frac{c}{3} \Theta^{ \pm},} \\
{\left[\bar{L}_{0}, \bar{\Theta}^{ \pm}\right]=\frac{\bar{c}}{6} \bar{\Theta}^{ \pm} \mp \bar{\Theta}^{ \pm} \circ \bar{J}_{0},} & {\left[\bar{J}_{0}, \bar{\Theta}^{ \pm}\right]=\mp \frac{\bar{c}}{3} \bar{\Theta}^{ \pm},}
\end{array}
$$

but with all other commutators vanishing. The fields associated to $\Theta^{ \pm} \Omega, \bar{\Theta}^{ \pm} \Omega$ by the state-field correspondence (Definition 3) are denoted $J^{ \pm}(z)$ and $\bar{J}^{ \pm}(\bar{z})$, respectively. By Proposition 1 the conformal field theoretic elliptic genus $\mathscr{E}(\tau, z)$ of such a CFT is a weak Jacobi form of weight 0 and index 1 . However, the space of such Jacobi forms is one-dimensional, as follows from the methods introduced in [EZ85] (see [BL00] or [Wen00, Thm. 3.1.12] for direct proofs). According to the discussion that follows Definition 7 the (complex) elliptic genus $\mathscr{E}_{\mathrm{K} 3}(\tau, z)$ of a K3 surface is a weak Jacobi form of weight 0 and index 1 as well, which by 18 is non-zero, since $\mathscr{E}_{\mathrm{K} 3}(\tau, z=0)=\chi(\mathrm{K} 3)=24$. The precise form of the function $\mathscr{E}_{\mathrm{K} 3}(\tau, z)$ is well-known, and we obtain 
Snapshots of Conformal Field Theory

$$
\mathscr{E}(\tau, z)=a \cdot \mathscr{E}_{\mathrm{K} 3}(\tau, z)=a \cdot\left(2 y+20+2 y^{-1}+\mathscr{O}(q)\right)
$$

for some constant $a$. In fact,

Proposition 2 ([Wen00, §7.1]). Consider an $N=(2,2)$ superconformal field theory at central charges $c=6, \bar{c}=6$ with space-time supersymmetry and such that all the eigenvalues of $J_{0}$ and of $\bar{J}_{0}$ are integral.

1. The elliptic genus of this CFT either vanishes, or it agrees with the complex elliptic genus $\mathscr{E}_{\mathrm{K} 3}(\tau, z)$ of a $\mathrm{K} 3$ surface.

2. The conformal field theoretic elliptic genus vanishes if and only if the theory is a toroidal $N=(2,2)$ superconformal field theory according to Definition 5 .

This result is mentioned in [NW01] and proved in [Wen00, §7.1], where the proof however contains a few typos. The sketch of a corrected proof is banned to the Appendix, since it uses a number of properties of superconformal field theories with space-time supersymmetry which are well-known to the experts, but which we have not derived in this exposition.

While as mentioned before, the toroidal $N=(2,2)$ superconformal field theories are well understood, it is also not hard to find examples of theories whose conformal field theoretic elliptic genus is $\mathscr{E}_{\mathrm{K} 3}(\tau, z)$, see [EOTY89]. In particular, the authors of [EOTY89] prove that the standard $\mathbb{Z}_{2}$-orbifold of every toroidal $N=(2,2)$ superconformal field theory at central charges $c=6, \bar{c}=6$ yields such an example. By the above this is in accord with the expectations based on the Kummer construction, hence our

Definition 8. A superconformal field theory is called a K3 THEORY, if the following conditions hold: the CFT is an $N=(2,2)$ superconformal field theory at central charges $c=6, \bar{c}=6$ with space-time supersymmetry, all the eigenvalues of $J_{0}$ and of $\bar{J}_{0}$ are integral, and the conformal field theoretic elliptic genus of the theory is

$$
\mathscr{E}(\tau, z)=\mathscr{E}_{\mathrm{K} 3}(\tau, z)
$$

Possibly, every K3 theory allows a non-linear sigma model interpretation on some K3 surface, however a proof is far out of reach. Nevertheless, under standard assumptions on the deformation theory of such theories it is possible to determine the form of every connected component of the moduli space of K3 theories. Namely, one assumes that all deformations by so-called marginal operators are integrable for these theories, an assumption which can be justified in string theory and which is demonstrated to all orders of perturbation theory in [Dix88]. Then, based on the previous results [Sei88, Cec91], one obtains 
Theorem 4 ([AM94, NW01]). With the notations introduced in Theorem 1 let $\mathscr{T}^{4,20}$ denote the Grassmannian of maximal positive definite oriented subspaces of $\mathbb{R}^{4,20}$,

$$
\mathscr{T}^{4,20}:=O^{+}(4,20 ; \mathbb{R}) / S O(4) \times O(20) .
$$

By $\mathscr{T}_{0}^{4,20} \subset \mathscr{T}^{4,20}$ we denote the set of all those maximal positive definite oriented subspaces $x \subset \mathbb{R}^{4,20}$ which have the property that $x^{\perp}$ does not contain any ROOTS, that is, all $\alpha \in x^{\perp} \cap \mathbb{Z}^{4,20}$ obey $\langle\alpha, \alpha\rangle \neq-2$.

If the above-mentioned assumptions on deformations of $K 3$ theories hold, namely that all deformations by so-called marginal operators are integrable, then each connected component $\mathscr{M}_{s}^{\mathrm{K} 3}$ of the moduli space of K3 theories has the following form:

$$
\mathscr{M}_{s}^{\mathrm{K} 3}=O^{+}(4,20 ; \mathbb{Z}) \backslash \mathscr{T}_{0}^{4,20}
$$

This result reinforces the expectation that one connected component $\mathscr{M}_{\sigma}^{\mathrm{K} 3}$ of the moduli space of $\mathrm{K} 3$ theories can be identified with the space of non-linear sigma models on $\mathrm{K} 3$ surfaces, since in addition, we have

Proposition 3 ([AM94]). The partial completion $\mathscr{T}^{4,20}$ of the smooth universal covering space $\mathscr{T}_{0}^{4,20}$ of $\mathscr{M}_{s}^{\mathrm{K} 3}$ can be isometrically identified with the PARAMETER SPACE OF NON-LINEAR SIGMA MODELS ON K3. Namely, denoting by $X$ the diffeomorphism type of a $K 3$ surface, $\mathscr{T}^{4,20}$ is a cover of the space of triples $(\Sigma, V, B)$ where $\Sigma$ denotes a HYPERKÄHLER STRUCTURE on $X, V \in \mathbb{R}^{+}$is interpreted as the VOLUME of $X$, and $B$ is the de Rham cohomology class of a real closed two-form on $X$, a so-called B-FIELD.

If a $K 3$ theory in $\mathscr{M}_{s}^{\mathrm{K} 3}$ lifts to a point in $\mathscr{T}^{4,20}$ which is mapped to the triple $(\Sigma, V, B)$, then $(\Sigma, V, B)$ is called a GEOMETRIC INTERPRETATION of the $K 3$ theory.

In [NW01, Wen01] it is shown that the expectation that non-linear sigma models on $\mathrm{K} 3$ yield $\mathrm{K} 3$ theories indeed is compatible with orbifold constructions, more precisely with every orbifold construction of a $\mathrm{K} 3$ surface from a complex twotorus by means of a discrete subgroup of $\mathrm{SU}(2)$. As mentioned above, one might conversely expect that every $\mathrm{K} 3$ theory with geometric interpretation $(\Sigma, V, B)$ can be constructed as a non-linear sigma model on a K3 surface, specified by the data $(\Sigma, V, B)$. At least the existence of a non-linear sigma model interpretation has not been disproved for any $\mathrm{K} 3$ theory, so far.

The statement of Proposition 3 makes use of the fact that every K3 surface is a hyperkähler manifold. The analogous statement for $\mathrm{K} 3$ theories is the observation that the two commuting copies of $N=2$ superconformal algebras (9)-(10) are each extended to an $N=4$ SUPERCONFORMAL ALGEBRA in these theories. This 
is a direct consequence of our Definition 8 of $\mathrm{K} 3$ theories. Indeed, as mentioned at the beginning of this section, the assumption of space-time supersymmetry together with the integrality of the eigenvalues of $J_{0}$ and $\bar{J}_{0}$ imply that the fields $J^{ \pm}(z), \bar{J}^{ \pm}(\bar{z})$ corresponding to two-fold left- and right-handed spectral flow are fields of the CFT. One checks that at central charges $c=6, \bar{c}=6$, these fields create states in the subspaces $W_{1}$ and $\bar{W}_{1}$ of the vectorspaces underlying the chiral algebras of Property C(see the vacuum axiom in Definition 3 ), whose $J_{0^{-}}$(respectively $\bar{J}_{0^{-}}$) eigenvalues are \pm 2 . Moreover, with the $U(1)$-currents $J(z), \bar{J}(\bar{z})$ of the two commuting copies of $N=2$ superconformal vertex algebras, the fields $J^{ \pm}(z), \bar{J}^{ \pm}(\bar{z})$ generate two commuting copies of a so-called $\mathfrak{s u}(2)_{1}$-CURRENT ALGEBRA, which in turn is known to extend the $N=2$ superconformal algebra to an $N=4$ superconformal algebra $\left[\mathrm{ABD}^{+} 76\right]$.

The characters of the irreducible unitary representations of the relevant $N=$ 4 superconformal algebra at arbitrary central charges have been determined in [ET87, ET88a, ET88b, ET88c, Ta090]. Their transformation properties under modular transforms in general are not modular, in contrast to the situation at lower supersymmetry, where an infinite class of characters of irreducible unitary representations does enjoy modularity. Instead, these $N=4$ characters exhibit a so-called Mock MODULAR behavior, see e.g. [DMZ] for a recent account. Since in the context of non-linear sigma models, $N=4$ supersymmetry is linked to the geometric concept of hyperkähler manifolds [AGF81], this seems to point towards a connection between Mock modularity and hyperkähler geometry. The nature of this connection however, to date, is completely mysterious.

\section{The elliptic genus of $\mathrm{K3}$}

Recall that the elliptic genus $\mathscr{E}_{\mathrm{K} 3}(\tau, z)$ of $\mathrm{K} 3$ plays center stage in our Definition 8 of $\mathrm{K} 3$ theories. Though this function is explicitly known and well understood, recent years have uncovered a number of mysteries around it. In the present section, some of these mysteries are discussed. This involves more open than solved problems, and as a reminder, the titles of all the following subsections are questions instead of statements.

\subsection{A non-geometric decomposition of the elliptic genus?}

As was mentioned at the end of Section 3, our very Definition 8 ensures that every K3 theory enjoys $N=(4,4)$ supersymmetry. The current section summarizes how this induces a decomposition of the function $\mathscr{E}_{\mathrm{K} 3}(\tau, z)$, which is a priori not motivated geometrically and which turns out to bear some intriguing surprises. 
In what follows, assume that we are given a K3 theory according to Definition 8 with space of states $\mathbb{H}=\mathbb{H}^{N S} \oplus \mathbb{H}^{R}$. Both $\mathbb{H}^{N S}$ and $\mathbb{H}^{R}$ can be decomposed into direct sums of irreducible unitary representations with respect to the $N=(4,4)$ superconformal symmetry. According to [ET87, ET88a], there are three types of irreducible unitary representations of the relevant $N=4$ superconformal algebra at central charge $c=6$, namely the VACUUM REPRESENTATION, the MASSLESS MATTER REPRESENTATION, and finally the MASSIVE MATTER REPRESENTATIONS which form a one-parameter family indexed by $h \in \mathbb{R}_{>0}$. For later convenience we focus on the Ramond-sector $\mathbb{H}^{R}$ of our theory and denote the respective irreducible unitary representations by $\mathscr{H}_{0}, \mathscr{H}_{\mathrm{mm}}, \mathscr{H}_{h}\left(h \in \mathbb{R}_{>0}\right)$. This notation alludes to the properties of the corresponding representations in the Neveu-Schwarz sector $\mathbb{H}^{N S}$, which are related to the representations in $\mathbb{H}^{R}$ by spectral flow $\Theta$ according to (13). Indeed, the vacuum representation in the NS-sector has the vacuum $\Omega$ as its ground state. The massive matter representations are characterized by the spontaneous breaking of supersymmetry at every mass level, including the ground state [Wit82].

Setting $y=\exp (2 \pi i z)$ and $q=\exp (2 \pi i \tau)$ for $z, \tau \in \mathbb{C}$ with $\mathfrak{I}(\tau)>0$ as before and using $c / 24=1 / 4$, the characters of the irreducible unitary $N=4$ representations that are relevant to our discussion are denoted by

$$
\chi_{a}(\tau, z):=\operatorname{Str}_{\mathscr{H}_{a}}\left(y^{J_{0}} q^{L_{0}-1 / 4}\right)=\operatorname{Tr}_{\mathscr{C}_{a}}\left((-1)^{J_{0}} y^{J_{0}} q^{L_{0}-1 / 4}\right), a \in \mathbb{R}_{\geq 0} \cup\{\mathrm{mm}\} .
$$

These functions have been determined explicitly in [ET88a] $]$. For our purposes, only the following properties are relevant,

$$
\begin{array}{rlrl}
\chi_{0}(\tau, z=0) & =-2, \quad \chi_{\mathrm{mm}}(\tau, z=0) & =1, \\
\forall h>0: \quad \chi_{h}(\tau, z) & =q^{h} \widehat{\chi}(\tau, z) \quad \text { with } \quad \widehat{\chi}(\tau, z) & =\chi_{0}(\tau, z)+2 \chi_{\mathrm{mm}}(\tau, z), \\
\text { hence } \quad \chi_{h}(\tau, z=0) & =\widehat{\chi}(\tau, z=0)=0 .
\end{array}
$$

The constant $\chi_{a}(\tau, z=0)$ yields the so-called WitTEn InDEX Wit82, Wit87, Wit88a] of the respective representation.

The most general ansatz for a decomposition of $\mathbb{H}^{R}$ into irreducible representations of the two commuting $N=4$ superconformal algebras therefore reads

$$
\mathbb{H}^{R}=\bigoplus_{a, \bar{a} \in \mathbb{R}_{\geq 0} \cup\{\mathrm{mm}\}} m_{a, \bar{a}} \mathscr{H}_{a} \otimes \overline{\mathscr{H}_{\bar{a}}}
$$

with appropriate non-negative integers $m_{a, \bar{a}}$. Then

$$
\operatorname{Tr}_{\mathbb{H}^{R}}\left((-1)^{J_{0}-\bar{J}_{0}} y^{J_{0}} \bar{y}^{\bar{J}_{0}} q^{L_{0}-1 / 4} \bar{q}^{\bar{L}_{0}-1 / 4}\right)=\sum_{a, \bar{a} \in \mathbb{R}_{\geq 0} \cup\{\mathrm{mm}\}} m_{a, \bar{a}} \cdot \chi_{a}(\tau, z) \cdot \overline{\chi_{\bar{a}}(\tau, z)},
$$

together with Definition 6yields the conformal field theoretic elliptic genus of our CFT as

$$
\mathscr{E}(\tau, z)=\sum_{a, \bar{a} \in \mathbb{R} \geq 0 \cup\{\mathrm{mm}\}} m_{a, \bar{a}} \cdot \chi_{a}(\tau, z) \cdot \overline{\chi_{\bar{a}}(\tau, z=0)} .
$$


This expression simplifies dramatically on insertion of 211. In addition, the known properties of K3 theories impose a number of constraints on the coefficients $m_{a, \bar{a}}$. First, since under spectral flow, $\mathscr{H}_{0}$ is mapped to the irreducible representation of the $N=4$ superconformal algebra whose ground state is the vacuum $\Omega$, the uniqueness of the vacuum (see Property C) implies $m_{0,0}=1$. Moreover, from the proof of Proposition 2 (see the Appendix) or from the known explicit form (20) of $\mathscr{E}(\tau, z)$, we deduce that in every $\mathrm{K} 3$ theory, $m_{0, \mathrm{~mm}}=m_{\mathrm{mm}, 0}=0$. Finally, according to the discussion of Property $\mathrm{B}$ in Section 2.2, $\mathbb{H}_{h, \bar{h}} \cap \mathbb{H}_{b}$ can only be non-trivial if $h-\bar{h} \in \mathbb{Z}$, which on $\mathbb{H}_{h, \bar{h}} \cap \mathbb{H}_{f} \cap \mathbb{H}^{N S}$ generalizes to $h-\bar{h} \in \frac{1}{2}+\mathbb{Z}$. Since the groundstates of $\mathscr{H}_{0}, \mathscr{H}_{\mathrm{mm}}, \mathscr{H}_{h}$ under spectral flow yield states with $L_{0}$ eigenvalues $0, \frac{1}{2}, h$, and $J_{0}$-eigenvalues $0, \pm 1,0$, respectively [ET88a], this implies that $m_{0, \bar{h}}, m_{\mathrm{mm}, \bar{h}}, m_{h, 0}, m_{h, \mathrm{~mm}}$ with $h, \bar{h}>0$ can only be non-zero if $h, \bar{h} \in \mathbb{N}$. In conclusion, we obtain a refined ansatz for the $N=(4,4)$ decomposition of $\mathbb{H}^{R}$,

$$
\begin{aligned}
\mathbb{H}^{R}=\mathscr{H}_{0} \otimes \overline{\mathscr{H}_{0}} \oplus h^{1,1} \mathscr{H}_{\mathrm{mm}} \otimes \overline{\mathscr{H}_{\mathrm{mm}}} \oplus \bigoplus_{h, \bar{h} \in \mathbb{R}_{>0}} k_{h, \bar{h}} \mathscr{H}_{h} \otimes \overline{\mathscr{H}_{\bar{h}}} \\
\oplus \bigoplus_{n=1}^{\infty}\left[f_{n} \mathscr{H}_{n} \otimes \overline{\mathscr{H}_{0}} \oplus \overline{f_{n}} \mathscr{H}_{0} \otimes \overline{\mathscr{H}_{n}}\right] \\
\oplus \bigoplus_{n=1}^{\infty}\left[g_{n} \mathscr{H}_{n} \otimes \overline{\mathscr{H}_{\mathrm{mm}}} \oplus \overline{g_{n}} \mathscr{H}_{\mathrm{mm}} \otimes \overline{\mathscr{H}_{n}}\right] .
\end{aligned}
$$

Here, all the coefficients $h^{1,1}, k_{h, \bar{h}}, f_{n}, \bar{f}_{n}, g_{n}, \bar{g}_{n}$ are non-negative integers, whose precise values depend on the specific K3 theory under inspection. By (22), and inserting (21) and the refined ansatz (23), we obtain

$$
\begin{aligned}
\mathscr{E}(\tau, z) & =-2 \chi_{0}(\tau, z)+h^{1,1} \chi_{\mathrm{mm}}(\tau, z)+\sum_{n=1}^{\infty}\left[-2 f_{n}+g_{n}\right] \chi_{n}(\tau, z) \\
& =-2 \chi_{0}(\tau, z)+h^{1,1} \chi_{\mathrm{mm}}(\tau, z)+e(\tau) \widehat{\chi}(\tau, z) \text { with } e(\tau):=\sum_{n=1}^{\infty}\left[g_{n}-2 f_{n}\right] q^{n} .
\end{aligned}
$$

Now recall from Definition 8 that $\mathscr{E}(\tau, z)=\mathscr{E}_{\mathrm{K} 3}(\tau, z)$, where by the discussion preceding (20) we have $\mathscr{E}_{\mathrm{K} 3}(\tau, z=0)=24$. Using (21), this implies $h^{1,1}=20$. Since the complex elliptic genus $\mathscr{E}_{\mathrm{K} 3}(\tau, z)$ is a topological invariant of all $\mathrm{K} 3$ surfaces, we conclude

Proposition 4. The elliptic genus $\mathscr{E}_{\mathrm{K} 3}(\tau, z)$ of $K 3$ decomposes into the characters of irreducible unitary representations of the relevant $N=4$ superconformal algebra in the Ramond sector according to

$$
\begin{aligned}
\mathscr{E}_{\mathrm{K} 3}(\tau, z)=-2 \chi_{0}(\tau, z)+ & 20 \chi_{\mathrm{mm}}(\tau, z)+e(\tau) \widehat{\chi}(\tau, z), \\
& \text { where } e(\tau):=\sum_{n=1}^{\infty}\left[g_{n}-2 f_{n}\right] q^{n},
\end{aligned}
$$


and the coefficients $g_{n}, f_{n}$ give the respective multiplicities of representations in the decomposition (23). While the values of $g_{n}, f_{n}$ vary within the moduli space of $K 3$ theories, the coefficients $g_{n}-2 f_{n}$ of $e(\tau)$ are invariant.

A decomposition of $\mathscr{E}_{\mathrm{K} 3}(\tau, z)$ in the spirit of Proposition 4 was already given in [EOTY89]. In [Oog89] and independently in [Wen00, Conj.7.2.2] it was conjectured that all coefficients of the function $e(\tau)$ are non-negative, for the following reason: recall that under spectral flow, the irreducible representation $\mathscr{H}_{0}$ is mapped to the representation of the $N=4$ superconformal algebra whose ground state is the vacuum $\Omega$. Therefore, in (23), the coefficients $f_{n}$ determine those contributions to the subspace $W_{n} \subset W$ of the vectorspace underlying the chiral algebra of Property that do not belong to the vacuum representation under the $N=(4,4)$ supersymmetry. For any fixed value of $n \in \mathbb{N}$ with $n>0$, we generically expect no such additional contributions to $W_{n}$. In other words, we expect that generically $f_{n}=0$ and thus that the $n^{\text {th }}$ coefficient of $e(\tau)$ agrees with $g_{n} \geq 0$. Since these coefficients are invariant on the moduli space of K3 theories, they should always be non-negative.

The conjectured positivity of the coefficients $g_{n}-2 f_{n}$ is proved in [EH09, EOT11] in the context of an intriguing observation. Namely, in [EOT11], Eguchi, Ooguri and Tachikawa observe that each of these coefficients seems to give the dimension of a representation of a certain sporadic group, namely of the MATHIEU GROUP $M_{24}$. For small values of $n$, they find dimensions of irreducible representations, while at higher order, more work is required to arrive at a well-defined conjecture. The quest for understanding this observation, which is often referred to as Mathieu Moonshine, has sparked enormous interest in the mathematical physics community. Building on results of [Che10, GHV10a, GHV10b, EH11], the observation has been recently verified by Gannon in the following form:

Theorem 5 ([Gan16]). There are virtual representations of the Mathieu group $M_{24}$ on spaces $\mathscr{R}_{0}, \mathscr{R}_{\mathrm{mm}}$, and true representations on spaces $\mathscr{R}_{n}$, $n \in \mathbb{N}_{>0}$, such that

$$
\mathscr{R}:=\mathscr{H}_{0} \otimes \mathscr{R}_{0} \oplus \mathscr{H}_{\mathrm{mm}} \otimes \mathscr{R}_{\mathrm{mm}} \oplus \bigoplus_{n=1}^{\infty} \mathscr{H}_{n} \otimes \mathscr{R}_{n}
$$

has the following properties: with the $N=4$ superconformal algebra acting non-trivially only on the first factor in each summand of $\mathscr{R}$, and the Mathieu group $M_{24}$ acting non-trivially only on the second factor, one obtains functions

$$
\forall g \in M_{24}: \quad \mathscr{E}_{g}(\tau, z):=\operatorname{Tr}_{\mathscr{R}}\left((-1)^{J_{0}} g y^{J_{0}} q^{L_{0}-1 / 4}\right)
$$

which under modular transformations generate a collection of $M_{24}$-TWISTED ELLIPTIC GENERA of K3. In particular, $\mathscr{E}_{\mathrm{id}}(\tau, z)=\mathscr{E}_{\mathrm{K} 3}(\tau, z)$. 


\subsection{A geometric Mathieu Moonshine phenomenon?}

While Theorem 5 beautifully specifies a well-defined formulation of the Mathieu Moonshine observation and proves it, the proof does not offer any insight into the role of the Mathieu group $M_{24}$ in the context of K3 theories. The present section summarizes some ideas for a possible interpretation that is based in geometry.

Indeed, the relevance of the group $M_{24}$ for the geometry of K3 surfaces had been discovered much earlier by Mukai:

Theorem 6 (|[Muk88]). Let $G$ denote a finite group of SYMPLECTIC AUTOMORPHISMS of a K3 surface $X$. By this we mean that $X$ denotes a $K 3$ surface whose complex structure has been fixed, and that $G$ is a finite group of biholomorphic maps on $X$ whose induced action on the holomorphic volume form is trivial.

Then $G$ is a subgroup of the Mathieu group $M_{24}$. More precisely, $G$ is a subgroup of one out of a list of 11 subgroups of $M_{23} \subset M_{24}$, the largest of which has order 960.

Hence although $M_{24}$ does play a crucial role in describing symplectic automorphisms of K3 surfaces, Theorem 6 cannot immediately explain Mathieu Moonshine. Indeed, Mathieu Moonshine suggests that there is an action of the entire group $M_{24}$ on some mathematical object which underlies the elliptic genus of K3, while Theorem 6 implies that no $\mathrm{K} 3$ surface allows $M_{24}$ as its symplectic automorphism group. Namely, the theorem states that the maximal order of a symplectic automorphism group of any K3 surface is 960, which is smaller by orders of magnitude than the order 244.823 .040 of $M_{24}$.

Since the non-geometric decomposition of $\mathscr{E}_{\mathrm{K} 3}(\tau, z)$ by means of $N=4$ supersymmetry presented in Section 4.1 led to the discovery of Mathieu Moonshine, one may suspect that rather than the properties of $\mathrm{K} 3$ surfaces, the properties of $\mathrm{K} 3$ theories should explain the Mathieu Moonshine phenomena. However, symmetry groups of K3 theories, in general, need not be subgroups of $M_{24}$, as apparently was first noted independently by the authors of [EOT11] and [TW13]. Conversely, no K3 theory can have $M_{24}$ as its symmetry group, as follows from [GHV12], where Gaberdiel, Hohenegger and Volpato generalize a very enlightening second proof of Theorem [6 due to Kondo [Kon98] to a classification result for symmetries of K3 theories.

Because by the above, the symmetries of $\mathrm{K} 3$ theories seem not to explain Mathieu Moonshine, in a series of papers [TW13, TW15b, TW15a], Taormina and the author have proposed constructions that may lead to the action of $M_{24}$ as a combined action of all finite symplectic automorphism groups of $\mathrm{K} 3$ surfaces. This idea can be motivated by the mathematical properties of the elliptic genus which were presented in Sections 2.4 and 2.5 . 
By Theorem 3 the complex elliptic genus $\mathscr{E}_{\mathrm{K} 3}(\tau, z)$ is recovered from the chiral de Rham complex $\Omega_{X}^{\text {ch }}$ of a K3 surface $X$ as its graded Euler characteristic, that is, as the supertrace of the appropriate operator on the sheaf cohomology $H^{*}\left(X, \Omega_{X}^{\mathrm{ch}}\right)$. In accord with [GM04, (2.1.3)], one can expect that every symplectic automorphism of a K3 surface $X$ induces an action on $H^{*}\left(X, \Omega_{X}^{\text {ch }}\right)$. Therefore, the sheaf cohomology $H^{*}\left(\mathrm{~K} 3, \Omega_{\mathrm{K} 3}^{\text {ch }}\right)$ of the chiral de Rham complex appears to be an excellent candidate for the desired mathematical object which both underlies the elliptic genus, and which carries actions of all finite symplectic automorphism groups of K3 surfaces, thus combining them to the action of a possibly larger group.

Note that according to Theorem 3 , there exists a natural structure of a supervertex algebra on $H^{*}\left(\mathrm{~K} 3, \Omega_{\mathrm{K} 3}^{\mathrm{ch}}\right)$. This additional structure on the mathematical object which underlies the elliptic genus is in complete accord with the implications of Theorem 5] Indeed, it was already conjectured in [GPRV13, GPV15], that Mathieu Moonshine is governed by some vertex algebra which carries an $M_{24}$-action, whose properties would immediately induce the modular transformation properties of the twisted elliptic genera of Theorem 5 As was argued in the discussion of Theorem 3. $H^{*}\left(X, \Omega_{X}^{\mathrm{ch}}\right)$ is moreover expected to be related to a non-linear sigma model on $X$, at least in an infinite volume limit, providing the desired link to $\mathrm{K} 3$ theories. Indeed, the required compatibility with an infinite volume limit might also explain the restriction to those symmetries of K3 theories which can be induced by some symplectic automorphism of finite order of a $\mathrm{K} 3$ surface, as seems to be the case for the generators of $M_{24}$ in Mathieu Moonshine.

Unfortunately, despite all its convincing properties promoting it to an excellent candidate to resolve Mathieu Moonshine, the vertex algebra structure of $H^{*}\left(X, \Omega_{X}^{\mathrm{ch}}\right)$ is notoriously hard to calculate, as are the precise properties of general non-linear sigma models on K3, even in an infinite volume limit. Therefore, these ideas remain conjectural, so far. Sadly, known alternative constructions for vertex algebras that underlie the elliptic genus and that are easier to calculate seem not to explain Mathieu Moonshine [CH14].

The above-mentioned mechanism of combining symplectic automorphism groups of distinct K3 surfaces to larger groups, as presented in [TW13, TW15b], yields the following result, which can be seen as evidence in favor of these ideas:

Proposition 5 ([TW15a]). Consider the "smallest massive" representation of $M_{24}$ that occurs in Theorem [5, that is, the representation on $\mathscr{R}_{1}$.

The space $\mathscr{R}_{1}$ is isomorphic to a certain vectorspace $V^{C F T}$ of states which is common to all $K 3$ theories that are obtained by a standard $\mathbb{Z}_{2}$-orbifold construction from a toroidal $N=(2,2)$ superconformal field theory. Moreover, on $V^{C F T}$, the combined action of all finite symplectic automorphism groups of Kummer surfaces induces a faithful action of the maximal subgroup $\mathbb{Z}_{2}^{4} \rtimes A_{8}$ of order 322.560 in $M_{24}$. The resulting representation on $V^{C F T}$ is equivalent to the representation of $\mathbb{Z}_{2}^{4} \rtimes A_{8}$ on $\mathscr{R}_{1}$ which is induced by restriction from $M_{24}$ to this subgroup. 
This result is the first piece of evidence in the literature for any trace of an $M_{24^{-}}$ action on a space of states of a K3 theory. It is remarkable that the CFT techniques produce precisely the representation of a maximal subgroup of $M_{24}$ which is predicted by Mathieu Moonshine according to the idea of "combining symplectic automorphism groups". Note that the group $\mathbb{Z}_{2}^{4} \rtimes A_{8}$ is not a subgroup of $M_{23}$, indicating that indeed $M_{24}$ rather than $M_{23}$ should be expected to be responsible for Mathieu Moonshine, despite Theorem 6 by which all finite symplectic automorphism groups of K3 surfaces are subgroups of $M_{23}$. This preference for $M_{24}$ to $M_{23}$ is in accord with the findings of [Gan16].

Encouraged by Proposition 5, one may hope that in an infinite volume limit, $V^{C F T}$ can be identified with a subspace of $H^{*}\left(\mathrm{~K} 3, \Omega_{\mathrm{K} 3}^{\mathrm{ch}}\right)$, inducing an equivalence of vertex algebras. Furthermore, by combining the action of $\mathbb{Z}_{2}^{4} \rtimes A_{8}$ with the action of finite symplectic automorphism groups of K3 surfaces which are not Kummer, one may hope to generate an action of the entire group $M_{24}$. Finally, one may hope that this result generalizes to the remaining representations on $\mathscr{R}_{n}, n>1$, found in Theorem 5 In conclusion, there is certainly much work left.

\subsection{A geometric decomposition of the elliptic genus?}

Even if the ideas presented in Section 4.2 prove successful, then so far, they give no indication for the reason for $M_{24}$-of all groups- to arise from the combined action of finite symplectic automorphism groups of $\mathrm{K} 3$ surfaces. Circumventing this intrinsic problem, the current section presents a simpler conjecture which can be formulated independently of Mathieu Moonshine. If true, however, it could serve as a step towards understanding Mathieu Moonshine.

Taking the idea seriously that there should be a purely geometric explanation for Mathieu Moonshine, one main obstacle to unraveling its mysteries is the lack of geometric interpretation for the non-geometric decomposition of $\mathscr{E}_{\mathrm{K} 3}(\tau, z)$ stated in Proposition 4, which is at the heart of the discovery of Mathieu Moonshine. Recall that the derivation of Proposition 4 rests on the identification 19) of the complex elliptic genus of a Calabi-Yau $D$-manifold $X$ with the conformal field theoretic elliptic genus of a CFT that is obtained from $X$ by a non-linear sigma model construction. We have incorporated this identification into our Definition 8 , and it is the motivation for decomposing the complex elliptic genus of $\mathrm{K} 3$ into the characters of irreducible unitary representations of the relevant $N=4$ superconformal algebra at central charge $c=6$. While the conformal field theoretic elliptic genus by Definition 6 is obtained as a trace over the space of states $\mathbb{H}^{R}$, the complex elliptic genus by Definition 7 is an analytic trace over a formal power series $\mathbb{E}_{q,-y}$ whose coefficients are holomorphic vector bundles on our $\mathrm{K} 3$ surface. The decomposition of the space of states $\mathbb{H}^{R}$ of every K3 theory by $N=(4,4)$ supersymmetry which was performed in Section 4.1 to derive Proposition 4 should accordingly be counterfeited by a decomposition of $\mathbb{E}_{q,-y}$. We thus expect 
Conjecture 1. Let $X$ denote a $\mathrm{K} 3$ surface with holomorphic tangent bundle $T:=T^{1,0} X$, and consider $\mathbb{E}_{q,-y}$ as in Definition 7 Furthermore, let $e(\tau)$ denote the function defined in Proposition 4 Then there are polynomials $p_{n}$, $n \in \mathbb{N}_{>0}$, such that

$$
\begin{gathered}
\qquad \mathbb{E}_{q,-y}=-\mathscr{O}_{X} \cdot \chi_{0}(\tau, z)-T \cdot \chi_{\mathrm{mm}}(\tau, z)+\sum_{n=1}^{\infty} p_{n}(T) \cdot q^{n} \widehat{\chi}(\tau, z), \\
\text { and } \left.e(\tau)=\sum_{n=1}^{\infty}\left(\int_{X} \operatorname{Td}(X) p_{n}(T)\right)\right) \cdot q^{n}, \\
\text { where } p_{n}(T)=\bigoplus_{k=0}^{N_{n}} \alpha_{k} T^{\otimes k} \text { if } p_{n}(x)=\sum_{k=0}^{N_{n}} \alpha_{k} x^{k}, \alpha_{k} \in \mathbb{Z}, \text { and where } T^{\otimes 0}=\mathscr{O}_{X} \\
\text { is understood. }
\end{gathered}
$$

If 197 is interpreted as a generalization of the McKean-Singer Formula, as indicated in the discussion of that equation, then Conjecture 1 can be viewed as a generalization of a LOCAL INDEX THEOREM [Pat71, Gil73, ABP73, Get83]. Note that the conjecture is formulated without even alluding to Mathieu Moonshine, so it may be of independent interest. If true, then for each $n \in \mathbb{N}_{>0}$, every finite symplectic automorphism group of a $\mathrm{K} 3$ surface $X$ naturally acts on $p_{n}(T)$, and one may hope that this will yield insight into the descent of this action to the representation of $M_{24}$ on $\mathscr{R}_{n}$ which was found in Theorem[5.

\section{Acknowledgements}

It is my pleasure to thank Ron Donagi for his hospitality and for many inspiring discussions on elliptic genera and Mathieu Moonshine which have greatly influenced this work. My sincere thanks also go to Scott Carnahan, since the final touches to this exposition have benefited much from my discussions with him.

Some of the material underlying this exposition arose from lecture courses that I presented at two summer schools, and I am grateful for the invitations to these events: cordial thanks go to the organizers Alexander Cardona, Sylvie Paycha, Andrés Reyes, Hernán Ocampo and the participants of the 8th Summer School on "Geometric, Algebraic and Topological Methods for Quantum Field Theory 2013" at Villa de Leyva, Columbia, as well as the organizers Martin Schlichenmaier, Pierre Bielavsky, Harald Grosse, Ryoichi Kobayashi, Armen Sergeev, Oleg Sheinman, Weiping Zhang and the participants of the ESI School and Conference "Geometry and Quantization GEOQUANT 2013" at Vienna, Austria, for creating such inspiring events.

This work is in part supported by my ERC Starting Independent Researcher Grant StG No. 204757-TQFT "The geometry of topological quantum field theories".

\section{Appendix: proof of Proposition 2 in Section 3}

The entire proof of Proposition 2 rests on the study of the +1 -eigenspace of the linear operator $\bar{J}_{0}$ on the subspace $\bar{W}_{1 / 2}$ of the vectorspace $\bar{W}$ underlying the chiral algebra. First, one shows that this eigenspace is either trivial or two-dimensional, 
and from this one deduces claim 1. of the proposition. One direction of claim 2. is checked by direct calculation, using the defining properties of toroidal $N=(2,2)$ superconformal field theories. To obtain the converse, one shows that $\mathscr{E}(\tau, z) \equiv 0$ implies that an antiholomorphic counterpart of the conformal field theoretic elliptic genus must vanish as well, from which claim 2. is shown to follow.

1. Assume that the space $\bar{W}_{1 / 2}$ contains an eigenvector of $\bar{J}_{0}$ with eigenvalue +1 . We denote the field associated to this state by $\bar{\psi}_{1}^{+}(\bar{z})$. The properties of the real structure on the space of states $\mathbb{H}$ of our CFT imply that there is a complex conjugate state with $\bar{J}_{0}$-eigenvalue -1 whose associated field we denote by $\bar{\psi}_{1}^{-}(\bar{z})$. The properties of unitary irreducible representations of the Virasoro algebra imply that these fields form a DIRAC FERMION (see the discussion around (15)), and that therefore $\bar{J}_{1}(\bar{z}):=\frac{1}{2}: \bar{\psi}_{1}^{+} \bar{\psi}_{1}^{-}:(\bar{z})$ is a $U(1)$-current as in Example1 in Section 2.1] By a procedure known as GKO-CONSTRUCTION [GKO85], one obtains $\bar{J}(\bar{z})=\bar{J}_{1}(\bar{z})+\bar{J}_{2}(\bar{z})$ for the field $\bar{J}(\bar{z})$ in the $N=2$ superconformal algebra (9)(10), and $\bar{J}_{k}(\bar{z})=i \partial \bar{H}_{k}(\bar{z})$ with $\bar{\psi}_{1}^{ \pm}(\bar{z})=c_{1}^{ \pm}: e^{ \pm i \bar{H}_{1}}:(\bar{z})$ with cocycle factors $c_{1}^{ \pm}$. The fields of twofold right-handed spectral flow, which by assumption are fields of the theory, are moreover given by $\bar{J}^{ \pm}(\bar{z})=c^{ \pm}: e^{ \pm i\left(\bar{H}_{1}+\bar{H}_{2}\right)}:(\bar{z})$ with cocycle factors $c^{ \pm}$. Their OPEs with the $\bar{\psi}_{1}^{ \pm}(\bar{z})$ yield an additional Dirac-fermion, with fields $\bar{\psi}_{2}^{ \pm}(\bar{z}):=c_{2}^{ \pm}: e^{ \pm i \bar{H}_{2}}:(\bar{z})$ in the CFT and further cocycle factors $c_{2}^{ \pm}$. This proves that the \pm 1 -eigenspaces of $\bar{J}_{0}$ on $\bar{W}_{1 / 2}$ each are precisely two-dimensional, since by the same argument no further Dirac fermions can be fields of the theory. Note that by definition, the corresponding states belong to the sector $\mathbb{H}_{f} \cap \mathbb{H}^{N S} \subset \mathbb{H}$ of the space of states of our theory.

In summary, the +1 -eigenspace of the linear operator $\bar{J}_{0}$ on $\bar{W}_{1 / 2}$ is either trivial or two-dimensional.

We now study the leading order contributions in the conformal field theoretic elliptic genus $\mathscr{E}(\tau, z)$ of our theory. From $(20)$ and by the very Definition 6 we deduce that $2 a y^{-1}$ counts states in the subspace $V \subset \mathbb{H}^{R}$ where $L_{0}, \bar{L}_{0}$ both take eigenvalue $\frac{c}{24}=\frac{1}{4}=\frac{\bar{c}}{24}$ and $J_{0}$ takes eigenvalue -1 . More precisely,

$$
2 a=\operatorname{Tr}_{V}\left((-1)^{J_{0}-\bar{J}_{0}}\right)=-\operatorname{Tr}_{V}\left((-1)^{\bar{J}_{0}}\right) .
$$

As follows from properties of the so-called CHIRAL RING, see e.g. Wen00, §3.1.1], a basis of $V$ is obtained by spectral flow $\Theta$ (see Ingredient IV in Section 2.2 from (i) the vacuum $\Omega$, (ii) the state whose corresponding field is $\bar{J}^{+}(\bar{z})$, and (iii) a basis of the +1 -eigenspace of the linear operator $\bar{J}_{0}$ on $\bar{W}_{1 / 2}$. Since according to (13), the eigenvalues of $\bar{J}_{0}$ after spectral flow to $V$ are (i) -1 , (ii) +1 , (iii) 0 , the above trace vanishes if the +1 -eigenspace of the linear operator $\bar{J}_{0}$ on $\bar{W}_{1 / 2}$ is two-dimensional, implying $2 a=0$, and if this eigenspace is trivial, then we obtain $2 a=2$.

In conclusion, the conformal field theoretic elliptic genus of our theory either vanishes, in which case the +1 -eigenspace of the linear operator $\bar{J}_{0}$ on $\bar{W}_{1 / 2}$ is created by two Dirac fermions, or $\mathscr{E}(\tau, z)=\mathscr{E}_{\mathrm{K} 3}(\tau, z)$. 
2. a. Using the details of toroidal $N=(2,2)$ superconformal field theories that are summarized in Section 2.3 one checks by a direct calculation that the conformal field theoretic elliptic genus of all such theories vanishes.

b. To show the converse, first observe that in our discussion of $N=(2,2)$ superconformal field theories, the two commuting copies of a superconformal algebra are mostly treated on an equal level. However, the Definition 6 breaks this symmetry, and

$$
\overline{\mathscr{E}}(\tau, z):=\operatorname{Tr}_{\mathbb{H}^{R}}\left((-1)^{J_{0}-\bar{J}_{0}} \bar{y}_{0} q^{L_{0}-c / 24} \bar{q}^{\bar{L}_{0}-\bar{c} / 24}\right)
$$

should define an equally important antiholomorphic counterpart of the conformal field theoretic elliptic genus. In our case, by the same reasoning as for $\mathscr{E}(\tau, z)$, it must yield zero or $\overline{\mathscr{E}} \mathrm{K} 3(\tau, z)$. Note that Proposition 1 implies that

$$
\mathscr{E}(\tau, z=0)=\overline{\mathscr{E}}(\tau, z=0)
$$

is a constant, which in fact is known as the WiTTEN INDEX Wit82, Wit87, Wit88a]. In particular, by (18) we have $\mathscr{E}_{\mathrm{K} 3}(\tau, z=0)=24$, hence $\mathscr{E}(\tau, z) \equiv 0$ implies $\overline{\mathscr{E}}(\tau, z) \equiv 0$. It remains to be shown that our theory is a toroidal theory according to Definition 5 in this case.

But Step 1. of our proof then implies that the +1 -eigenspace of the linear operator $\bar{J}_{0}$ on $\bar{W}_{1 / 2}$ is created by two Dirac fermions and that the analogous statement holds for the +1 -eigenspace of the linear operator $J_{0}$ on $W_{1 / 2}$. Hence we have Dirac fermions $\psi_{k}^{ \pm}(z)$ and $\bar{\psi}_{k}^{ \pm}(\bar{z}), k \in\{1,2\}$, with OPEs as in (15). Compatibility with supersymmetry then implies that the superpartners of these fields yield the two $\mathfrak{u}(1)^{4}$-current algebras, as is required in order to identify our theory as a toroidal one.

\section{References}

ABD ${ }^{+}$76. M. Ademollo, L. Brink, A. D'Adda, R. D'Auria, E. Napolitano, S. Sciuto, E. Del Giudice, P. Di Vecchia, S. Ferrara, F. Gliozzi, R. Musto, AND R. PETTORINO, Supersymmetric strings and color confinement, Phys. Lett. B62 (1976), 105-110.

ABP73. M. ATIYAh, R. BotT, AND V.K. PATod, On the heat equation and the index theorem, Invent. Math. 19 (1973), 279-330, Errata: Invent. Math. 28, 277-280 (1975).

AGF81. L. AlVAREZ-GAUMÉ AND D.Z. FREEDMAN, Geometrical structure and ultraviolet finiteness in the supersymmetric $\sigma$-model, Commun. Math. Phys. 80 (1981), no. 3, 443-451.

AKMW87. O. Alvarez, T.P. Killingback, M. Mangano, And P. Windey, String theory and loop space index theorems, Commun. Math. Phys. 111 (1987), 1-12.

AM94. P.S. ASPINwall And D.R. MorRISOn, String theory on K3 surfaces, in: Mirror symmetry II, B. Greene and S.T. Yau, eds., AMS, 1994, pp. 703-716; arXiv: hep-th/9404151.

AS63. M.F. ATIYAH AND I.M. SINGER, The index of elliptic operators on compact manifolds, Bulletin American Mathematical Society 69 (1963), 322-433. 
BFK86. W. BOUCHER, D. FriedAn, AND A. Kent, Determinant formulae and unitarity for the $N=2$ superconformal algebras in two dimensions or exact results on string compactification, Phys. Lett. B172 (1986), 316-322.

BhPvdV04. W. Barth, K. Hulek, C. Peters, and A. van de Ven, Compact Complex Surfaces, Second enlarged ed., Springer-Verlag, Berlin Heidelberg, 2004.

BHS08. D. Ben-Zvi, R. Heluani, And M. SzCZESny, Supersymmetry of the chiral de Rham complex, Compos. Math. 144 (2008), no. 2, 503-521; arXiv: math/0601532 [math.QA].

BL00. L.A. BORISOV AND A. LIBGOBER, Elliptic genera of toric varieties and applications to mirror symmetry, Invent. Math. 140 (2000), no. 2, 453-485; arXiv: math/9904126 [math.AG].

BL03. ㄴ. Elliptic genera of singular varieties, Duke Math. J. 116 (2003), no. 2, 319351; arXiv: math/0007108 [math.AG].

Bor86. R.E. BORCHERDS, Vertex algebras, Kac-Moody algebras and the monster, Proc. Nat. Acad. Sci. U.S.A. 83 (1986), 3068-3071.

Bor01. L.A. BoRISOv, Vertex algebras and mirror symmetry, Commun. Math. Phys. 215 (2001), no. 2, 517-557; arXiv: math/9809094 [math.AG].

BPZ84. A.A. BELAVIN, A.M. Polyakov, AND A.B. ZAMOlodChiKov, Infinite conformal symmetry in two-dimensional quantum field theory, Nucl. Phys. B241 (1984), 333-380.

Car86. J.L. CARDY, Operator content of two-dimensional conformally invariant theories, Nucl. Phys. B270 (1986), no. 2, 186-204.

CdGP91. P. Candelas, X.C. De la Ossa, P.S. Green, and L. Parkes, A pair of Calabi-Yau manifolds as an exactly soluble superconformal theory, Nucl. Phys. B359 (1991), 21-74.

Cec91. S. CeCotTi, $N=2$ Landau-Ginzburg vs. Calabi-Yau $\sigma$-models: Non-perturbative aspects, Int. J. Mod. Phys. A6 (1991), 1749-1813.

Cent85. A. Casher, F. Englert, H. Nicolai, And A. TAOrmina, Consistent superstrings as solutions of the $D=26$ bosonic string theory, Phys. Lett. B162 (1985), 121-126.

CH14. Th. Creutzig And G. HöHn, Mathieu Moonshine and the Geometry of K3 Surfaces, Commun. Number Theory Phys. 8 (2014), no. 2, 295-328; arXiv: 1309.2671 [math.QA].

Che10. M.C.N. Cheng, $K 3$ surfaces, $N=4$ dyons, and the Mathieu group $M_{24}$, Commun. Number Theory Phys. 4 (2010), 623-657; arXiv: 1005.5415 [hep-th] .

CLS90. P. CANDElas, M. LYNKer, AND R. SChimmrigk, Calabi-Yau manifolds in weighted P(4), Nucl. Phys. B341 (1990), 383-402.

CN79. J.H. CONWAY AND S.P. NORTON, Monstrous moonshine, Bull. London Math. Soc. 11 (1979), no. 3, 308-339.

Dix88. L.J. DIXON, Some world-sheet properties of superstring compactifications, on orbifolds and otherwise, in: Superstrings, unified theories and cosmology 1987 (Trieste, 1987), vol. 4 of ICTP Ser. Theoret. Phys., World Sci. Publ., Teaneck, NJ, 1988, pp. 67-126.

DMVV97. R. DiJKGRAaF, G. MoORe, E. Verlinde, AND H. Verlinde, Elliptic genera of symmetric products and second quantized strings, Commun. Math. Phys. 185 (1997), 197-209.

DMZ. A. DABholkar, S. Murthy, AND D. ZAGier, Quantum black holes, wall crossing and mock modular forms; arXiv: 1208.4074 [hep-th] .

DPYZ86. P. Di VecChiA, J.L. Petersen, M. YU, AND H.B. ZHENG, Explicit construction of unitary representations of the $N=2$ superconformal algebra, Phys. Lett. B174 (1986), 280-295.

DY93. P. Di FRANCESCO AND S. YANKIELOWICZ, Ramond sector characters and $N=2$ Landau-Ginzburg models, Nucl. Phys. B409 (1993), 186-210; arXiv: hep-th/9305037. 
EH09. T. Eguchi AND K. HikAmi, Superconformal Algebras and Mock Theta Functions 2. Rademacher Expansion for K3 Surface, Commun. Number Theory Phys. 3 (2009), 531-554; arXiv: 0904.0911 [math-ph].

EH11. $\quad-$, Note on Twisted Elliptic Genus of K3 Surface, Phys. Lett. B694 (2011), 446455; arXiv: 1008.4924 [hep-th].

EOT11. T. Eguchi, H. Ooguri, AND Y. TACHIKAWA, Notes on the K3 surface and the Mathieu group $M_{24}$, Exp. Math. 20 (2011), no. 1, 91-96; arXiv: 1004.0956 [hep-th].

EOTY89. T. EGUChI, H. OOguri, A. TAORMina, AND S.-K. YANG, Superconformal algebras and string compactification on manifolds with $S U(n)$ holonomy, Nucl. Phys. B315 (1989), 193-221.

ET87. T. EGUCHI AND A. TAORMINA, Unitary representations of the $N=4$ superconformal algebra, Phys. Lett. B196 (1987), 75-81.

ET88a. Character formulas for the $N=4$ superconformal algebra, Phys. Lett. B200 (1988), 315-322.

ET88b. Lxtended superconformal algebras and string compactifications, Trieste School 1988: Superstrings, pp. 167-188.

ET88c. On the unitary representations of $N=2$ and $N=4$ superconformal algebras, Phys. Lett. 210 (1988), 125-132.

EY90. T. EGUCHI AND S.-K. YANG, N=2 superconformal models as topological field theories, Mod. Phys. Lett. A5 (1990), 1693-1701.

EZ85. M. EICHLER AND D. ZAGIER, The theory of Jacobi forms, Progress in Mathematics, vol. 55, Birkhäuser, Boston-Basel-Stuttgart, 1985.

FBZ04. E. FRENKEL AND D. BEN-Zvi, Vertex algebras and algebraic curves, second ed., Mathematical Surveys and Monographs, vol. 88, American Mathematical Society, Providence, RI, 2004.

FFK89. G. FELDER, J. FRÖHLICH, AND G. KELLER, On the structure of unitary conformal field theory. I. Existence of conformal blocks, Commun. Math. Phys. 124 (1989), no. 3, 417-463.

FG94. J. FRÖHLICH AND K. GAWẸDZKI, Conformal field theory and geometry of strings, in: Mathematical quantum theory. I. Field theory and many-body theory (Vancouver, BC, 1993), Amer. Math. Soc., Providence, RI, 1994, pp. 57-97; arXiv: hep-th/9310187.

FK81. I.B. FRENKEL AND V.G. KAC, Basic representations of affine Lie algebras and dual resonance models, Invent. Math. 62 (1980/81), no. 1, 23-66.

FKRW95. E. Frenkel, V. KAC, A. RADUL, AND W. WANG, $W(1+\infty)$ and $W(g l(N))$ with central charge $N$, Commun. Math. Phys. 170 (1995), no. 2, 337-357.

FLM84. I. FRENKEL, J. LEPOWSKY, AND A. MEURMAN, A natural representation of the Fischer-Griess Monster with the modular function J as character, Proc. Nat. Acad. Sci. U.S.A. 81 (1984), no. 10, Phys. Sci., 3256-3260.

FS07. E. FRENKEL AND M. SZCZESNY, Chiral de Rham complex and orbifolds, J. A1gebr. Geom. 16 (2007), no. 4, 599-624; arXiv: math/0307181 [math. AG] .

Gan06. T. GANNON, Moonshine beyond the Monster - The bridge connecting algebra, modular forms and physics, Cambridge Monographs on Mathematical Physics, Cambridge University Press, Cambridge, 2006.

Gan16. - Much ado about Mathieu, Adv. Math. 301 (2016), 322-358; arXiv: 1211.5531 [math.RT].

Get83. E. GETZLER, Pseudodifferential operators on supermanifolds and the Atiyah-Singer index theorem, Commun. Math. Phys. 92 (1983), no. 2, 163-178.

GHV10a. M.R. GAberdiel, S. HohenegGer, AND R. Volpato, Mathieu moonshine in the elliptic genus of K3, JHEP 1010 (2010), 062; arXiv: 1008.3778 [hep-th].

GHV10b. Mathieu twining characters for K3, JHEP 1009 (2010), 058; arXiv: 1006.0221 [hep-th]. 
GHV12. - Symmetries of K3 sigma models, Commun. Number Theory Phys. 6 (2012), 1-50; arXiv: 1106.4315 [hep-th].

Gil73. P.B. GiLkey, Curvature and the eigenvalues of the Dolbeault complex for Kaehler manifolds, Adv. Math. 11 (1973), 311-325.

Gin88. P. Ginsparg, Applied conformal field theory, Lectures given at the Les Houches Summer School in Theoretical Physics 1988 (Les Houches, France), pp. 1-168.

GKO85. P. GODDARD, A. KENT, AND D. Olive, Virasoro algebra and coset space models, Phys. Lett. B152 (1985), 88-101.

GKO86. Unitary representations of the Virasoro and super-Virasoro algebras, Commun. Math. Phys. 103 (1986), 105-119.

GM04. V. Gorbounov AND F. Malikov, Vertex algebras and the LandauGinzburg/Calabi-Yau correspondence, Mosc. Math. J. 4 (2004), no. 3, 729-779; arXiv: math.AG/0308114.

GP90. B.R. Greene And M.R. Plesser, Duality in Calabi-Yau moduli space, Nucl. Phys. B338 (1990), 15-37.

GPRV13. M.R. Gaberdiel, D. Persson, H. Ronellenfitsch, and R. Volpato, Generalised Mathieu Moonshine, Commun. Number Theory Phys. 7 (2013), no. 1, 145223; arXiv: 1211.7074 [hep-th].

GPV15. M.R. Gaberdiel, D. Persson, and R. Volpato, Generalised Moonshine and Holomorphic Orbifolds, in: String-Math 2012. Proceedings of the conference, Universität Bonn, Bonn, Germany, July 16-21, 2012, Providence, RI: American Mathematical Society (AMS), 2015, pp. 73-86; arXiv: 1302.5425 [hep-th] .

Hir54. F. HIRZEBRUCH, Arithmetic genera and the theorem of Riemann-Roch for algebraic varieties., Proc. Nat. Acad. Sci. U.S.A. 40 (1954), 110-114.

Hir66. F. HIRZEBRUCH, Topological methods in algebraic geometry, Grundl. Math. Wiss., vol. 131, Springer-Verlag, Heidelberg, 1966.

Hir88. Elliptic genera of level $N$ for complex manifolds, in: Differential geometric methods in theoretical physics, K. Bleuler and M. Werner, eds., Kluwer Acad. Publ., 1988, pp. 37-63.

jMS67. H.P. JUN. MCKEAN AND I.M. SINGER, Curvature and the eigenvalues of the Laplacian., J. of Diff. Geometry 1 (1967), 43-69.

Kac98. V.G. KAC, Vertex algebras for beginners, second ed., University Lecture Series, vol. 10, American Mathematical Society, Providence, RI, 1998.

Kap05. A. KAPUSTIN, Chiral de Rham complex and the half-twisted sigma-model; arXiv: hep-th/0504074.

KO03. A. KAPUSTIN AND D. ORLOV, Vertex algebras, mirror symmetry, and D-branes: the case of complex tori, Commun. Math. Phys. 233 (2003), no. 1, 79-136; arXiv: hep-th/0010293.

Kon98. S. Kondo, Niemeier lattices, Mathieu groups and finite groups of symplectic automorphisms of K3 surfaces, Duke Math. J. 92 (1998), 593-603, Appendix by S. Mukai.

Kri90. I. KRICHEVER, Generalized elliptic genera and Baker-Akhiezer functions, Math. Notes 47 (1990), 132-142.

Lan88. P.S. LANDWEBER, Elliptic curves and modular forms in algebraic topology, Lecture Notes in Math., vol. 1326, Springer-Verlag, Berlin, 1988.

LL07. B.H. LiAn AND A.R. Linshaw, Chiral equivariant cohomology. I., Adv. Math. 209 (2007), no. 1, 99-161; arXiv: math/0501084 [math.DG] .

LVW89. W. LERCHE, C. VAFA, AND N.P. WARNER, Chiral rings in $N=2$ superconformal theories, Nucl. Phys. B324 (1989), 427-474.

LW78. J. LEPOWSKY AND R.L. Wilson, Construction of the affine Lie algebra $A_{1}(1)$, Commun. Math. Phys. 62 (1978), no. 1, 43-53.

Man98. Y. MANin, Presentation of the Fields Medals and a special tribute, IMU Bulletin 43, October (1998). 
MSV99. F. Malikov, V. Schechtman, And A. Vaintrob, Chiral de Rham complex, Commun. Math. Phys. 204 (1999), no. 2, 439-473; arXiv: math/9803041 [math. AG].

Muk88. S. MUKAI, Finite groups of automorphisms of K3 surfaces and the Mathieu group, Invent. Math. 94 (1988), 183-221.

Nah91. W. NAHM, A proof of modular invariance, Int. J. Mod. Phys. A6 (1991), no. 16, $2837-2845$.

Nar86. K.S. NARAIN, New heterotic string theories in uncompactified dimensions $<10$, Phys. Lett. 169B (1986), 41-46.

NW01. W. NAhm AND K. Wendland, A hiker's guide to $K 3-$ Aspects of $N=(4,4)$ superconformal field theory with central charge $c=6$, Commun. Math. Phys. 216 (2001), 85-138; arXiv: hep-th/9912067.

Oog89. H. OogurI, Superconformal Symmetry and Geometry of Ricci Flat Kahler Manifolds, Int. J. Mod. Phys. A4 (1989), 4303-4324.

OS73. K. OSTERWALDER AND R. SCHRADER, Axioms for Euclidean Green's functions, Commun. Math. Phys. 31 (1973), 83-112.

OS75. Axioms for Euclidean Green's functions. II, Commun. Math. Phys. 42 (1975), 281-305, With an appendix by Stephen Summers.

Pat71. V.K. PATODI, An analytic proof of Riemann-Roch-Hirzebruch theorem for Kaehler manifolds, J. of Diff. Geometry 5 (1971), 251-283.

Qiu87. Z. QIU, Modular invariant partition functions for $N=2$ superconformal field theories, Phys. Lett. B198 (1987), 497-502.

Sch08. M. SCHOTTENLOHER, A mathematical introduction to conformal field theory, 2nd ed., Lecture Notes in Physics, vol. 759, Springer-Verlag, Berlin, 2008.

Sei88. N. SEIBERG, Observations on the moduli space of superconformal field theories, Nucl. Phys. B303 (1988), 286-304.

Tao90. A. TAORMINA, The $N=2$ and $N=4$ superconformal algebras and string compactifications, Mathematical physics (Islamabad, 1989), World Sci. Publishing, pp. 349370.

Tho79a. J.G. Thompson, Finite groups and modular functions, Bull. London Math. Soc. 11 (1979), no. 3, 347-351.

Tho79b. Some numerology between the Fischer-Griess Monster and the elliptic modular function, Bull. London Math. Soc. 11 (1979), no. 3, 352-353.

TW13. A. TAORMINA AND K. WENDLAND, The overarching finite symmetry group of Kummer surfaces in the Mathieu group M24, JHEP 08 (2013), 125; arXiv: 1107.3834 [hep-th].

TW15a. Symmetry-surfing the moduli space of Kummer K3s, Proc. of the Conference String-Math 2012, Proceedings of Symposia in Pure Mathematics, no. 90, pp. 129153; arXiv: 1303.2931 [hep-th].

TW15b. - A twist in the M24 moonshine story, Confluentes Mathematici 7 (2015), 83113; arXiv: 1303.3221 [hep-th].

Was95. A.J. WASSERMANN, Operator algebras and conformal field theory, Proceedings of the International Congress of Mathematicians, Vol. 1, 2 (Zürich, 1994) (Basel), Birkhäuser, pp. 966-979.

Wen. K. WENDLAND, Conformal field theory for mathematicians, book manuscript based on lecture notes for an MSc course taught by the author, currently 255 pages; preliminarily accepted for publication in the AMS-Series "Graduate Studies in Mathematics".

Wen00. Moduli spaces of unitary conformal field theories, Ph.D. thesis, University of Bonn, 2000, supervisor: W. Nahm; available on request.

Wen01. Consistency of orbifold conformal field theories on K3, Adv. Theor. Math. Phys. 5 (2001), no. 3, 429-456; arXiv: hep-th/ 0010281.

Wen10. On the geometry of singularities in quantum field theory, Proceedings of the International Congress of Mathematicians, Hyderabad, August 19-27, 2010, Hindustan Book Agency, pp. 2144-2170. 
Wit82. E. Witten, Constraints on supersymmetry breaking, Nucl. Phys. B202 (1982), 253316.

Wit87. Elliptic genera and quantum field theory, Commun. Math. Phys. 109 (1987), 525-536.

Wit88a. The index of the Dirac operator in loop space, in: Elliptic curves and modular forms in algebraic geometry, P. Landweber, ed., Springer-Verlag, 1988, pp. 161-181.

Wit88b. Topological sigma models, Commun. Math. Phys. 118 (1988), 411-449.

Wit92. —u Mirror manifolds and topological field theory, in: Essays on mirror manifolds, Internat. Press, Hong Kong, 1992, pp. 120-158; arXiv: hep-th/9112056.

Wit94. $\quad$ On the Landau-Ginzburg description of $N=2$ minimal models, Int. J. Mod. Phys. A9 (1994), 4783-4800; arXiv: hep-th/9304026.

ZF86. A.B. ZAMOlodchikov AND V.A. FATEeV, Disorder fields in two-dimensional conformal quantum field theory and $N=2$ extended supersymmetry, Sov. Phys. JETP 63 (1986), 913-919.

Zhu96. Y. ZHU, Modular invariance of characters of vertex operator algebras, J. Amer. Math. Soc. 9 (1996), 237-302. 